LBL-36086

UC-400

\title{
A TOUGH2 Equation-of-State Module for the Simulation of Two-Phase Flow of Air, Water, and a Miscible Gelling Liquid
}

\author{
S. Finsterle, G. J. Moridis, and K. Pruess
}

\author{
Earth Sciences Division \\ Lawrence Berkeley Laboratory \\ University of California \\ Berkeley, CA 94720
}

May 1994

This work is supported by the In Situ Remediation Technology Development Integrated Program, Office of Technology Development, Office of Environmental Restoration and Waste Management, U.S. Department of Energy, under contract DE-AC03-76SF00098.

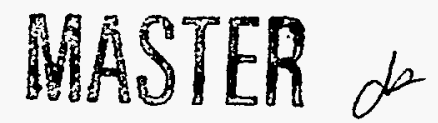

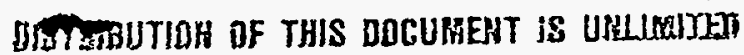




\section{-}

(2) 


\section{DISCLAIMER}

This report was prepared as an account of work sponsored by an agency of the United States Government. Neither the United States Government nor any agency thereof, nor any of their employees, make any warranty, express or implied, or assumes any legal liability or responsibility for the accuracy, completeness, or usefulness of any information, apparatus, product, or process disclosed, or represents that its use would not infringe privately owned rights. Reference herein to any specific commercial product, process, or service by trade name, trademark, manufacturer, or otherwise does not necessarily constitute or imply its endorsement, recommendation, or favoring by the United States Government or any agency thereof. The views and opinions of authors expressed herein do not necessarily state or reflect those of the United States Government or any agency thereof. 


\section{DISCLAIMER}

Portions of this document may be illegible in electronic image products. Images are produced from the best available original document. 


\section{AbSTRACT}

The injection of grout into the subsurface can be used to encapsulate contaminated regions of an aquifer, or to form underground barriers for the isolation of contaminant sources and to prevent the spreading of existing plumes. This requires identifying grouts, or barrier fluids, which when injected into the subsurface exhibit a large increase in viscosity and eventually solidify, sealing the permeable zones in the aquifer. Simulation and modeling analysis are indispensable tools for designing the injection and predicting the performance of the barrier. In order to model flow and transport in such systems, the thermophysical properties of the fluid mixtures have to be provided, and the governing mass- and energybalance equations for multiphase flow in porous media have to be solved numerically. The equation-of-state module EOS11 described herein is an extension of the EOS7 module of the TOUGH2 code for flow of saline water and air. In our modeling approach, the chemical grout is treated as a miscible fluid the viscosity of which is a function of time and concentration of the gelling agent in the pore water. If a certain high viscosity is reached and the movement of the grout plume ceases, the gel is assumed to solidify, leading to a new porous medium with changed soil characteristics, i.e. reduced porosity and permeability, increased capillary strength for a given water content, and changed initial saturation distribution. 


\section{TABLE OF CONTENTS}

Abstract $\ldots \ldots \ldots \ldots \ldots \ldots \ldots \ldots \ldots \ldots \ldots \ldots \ldots \ldots \ldots$

Table of Contents $\ldots \ldots \ldots \ldots \ldots \ldots \ldots \ldots \ldots \ldots \ldots \ldots \ldots$ iv

List of Figures $\ldots \ldots \ldots \ldots \ldots \ldots \ldots \ldots \ldots \ldots \ldots \ldots \ldots \ldots$

List of Tables $\ldots \ldots \ldots \ldots \ldots \ldots \ldots \ldots \ldots \ldots \ldots \ldots \ldots$

1. Introduction $\ldots \ldots \ldots \ldots \ldots \ldots \ldots \ldots \ldots \ldots \ldots \ldots \ldots \ldots \ldots$

2. Modeling approach $\ldots \ldots \ldots \ldots \ldots \ldots \ldots \ldots \ldots \ldots \ldots \ldots \ldots \ldots$

2.1 Process description. . . . . . . . . . . . . . . . . . 2

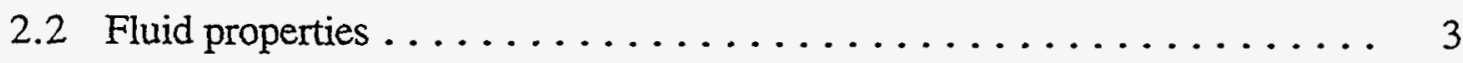

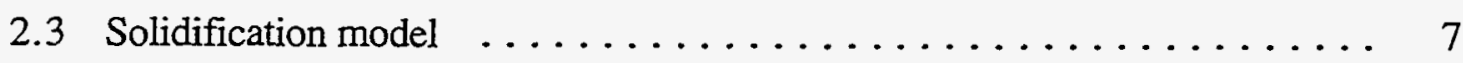

3. Using the gelation module EOS11 ... . . . . . . . . . . . 10

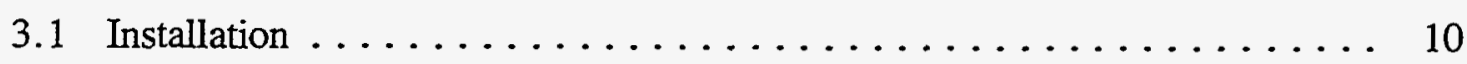

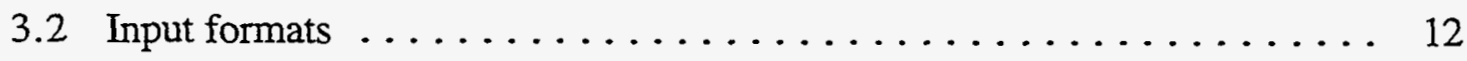

3.3 Application of Solidification Model $\ldots \ldots \ldots \ldots \ldots \ldots \ldots$

4. Sample problems $\ldots \ldots \ldots \ldots \ldots \ldots \ldots \ldots \ldots \ldots \ldots \ldots$

4.1 Sample 1: Multiple grout injections into unsaturated soil . . . . . . . . 18

4.2 Sample 2: Horizontal barrier emplacement ................ 29

Acknowledgment $\quad \ldots \ldots \ldots \ldots \ldots \ldots \ldots \ldots \ldots \ldots \ldots \ldots \ldots \ldots \ldots \ldots$

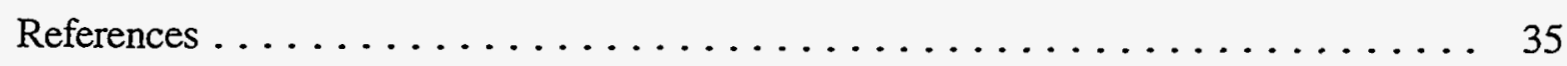

Appendix A: Subroutine GELLING . . . . . . . . . . . . . 37

Appendix B: Program SOLIMOD .................... 39 


\section{LIST OF FIgURES}

Figure 1: Viscosity as a function of gel concentration and time $\ldots \ldots \ldots \ldots$

Figure 2: Summary of EOS11 specifications and options . . . . . . . . . . 11

Figure 3: Schematic of sandbox and injected grout for sample problem No. $1 \ldots \ldots$

Figure 4: TOUGH2 input file for sample problem No. $1 \quad \ldots \ldots \ldots \ldots \ldots \ldots$

Figure 5: ITOUGH2 input file for sample problem No. $1 \quad \ldots \ldots \ldots \ldots \ldots \ldots$

Figure 6: Sample problem 1: Liquid saturation after preflushing . . . . . . . . . 21

Figure 7: Sample problem 1: Grout content immediately after primary injection . . . 22

Figure 8: Sample problem 1: Grout content at $t=5$ hours . . . . . . . . . 22

Figure 9: Sample problem 1: Porosity distribution after first solidification . . . . . . 23

Figure 10: Sample problem 1: Log-Permeability distribution after first solidification . . 23

Figure 11: Sample problem 1: Grout content immediately after second injection . . . . 25

Figure 12: Sample problem 1: Grout content at $t=10$ hours . . . . . . . . . . 25

Figure 13: Sample problem 1: Porosity distribution after second solidification . . . . 26

Figure 14: Sample problem 1: Log-Permeability distribution after second solidification . 26

Figure 15: Sample problem 1: Output prior to second solidification $\ldots \ldots \ldots \ldots 27$

Figure 16: Sample problem 1: Output after second solidification $\ldots \ldots \ldots \ldots$

Figure 17: TOUGH2 input file for sample problem No. $2 \ldots \ldots \ldots \ldots$

Figure 18: Sample problem 2: Permeability distribution . . . . . . . . . . . 31

Figure 19: Sample problem 2: Grout concentration after 6 hours . . . . . . . . . 32

Figure 20: Sample problem 2: Grout concentration after 1 day $\ldots \ldots \ldots . \ldots 32$

Figure 21: Sample problem 2: Grout concentration after 2 days . . . . . . . . 33

Figure 22: Sample problem 2: Grout concentration after 3 days . . . . . . . . 33

Figure 23: Sample problem 2: Permeability field of grouted aquifer $\ldots \ldots \ldots$. . . 34

\section{LIST OF TABLES}

Table 1: $\quad$ Concept and application of Solidification Model $\ldots \ldots \ldots \ldots$ 


\section{INTRODUCTION}

The injection of grout into the subsurface is a technique used to control the flow of contaminated groundwater. For example, injection of liquids with increasing viscosity can be used to encapsulate pollutant, to emplace underground barriers for containment of contaminant sources, and to prevent the spread of existing plumes. The processes responsible for the increase of viscosity may be manifold. Colloidal silica suspensions, for example, undergo a gelling process if the ionic strength of the solution is increased by adding a saline solution. Mixing of two polymeric systems, such as polysiloxenes, induces crosslinking and produces rubber-like networks of molecules. Fluids with strongly temperature-dependent viscosity solidify as they cool after being injected at high temperatures. Polybutenes or paraffin waxes are examples of the last group of potential barrier fluids.

When grout is injected into unsaturated soils, the grout plume slumps under gravity and spreads due to capillary forces, leaving the soil only partially saturated, thus achieving a lower permeability reduction upon gelling compared to the fully clogged pore space. The numerical model used to study the emplacement of barrier fluids in the vadose zone must account for the increase of the viscosity of the grout-water mixtures, and be able to simulate multiphase flow effects. TOUGH2 [Pruess, 1987, 1991a] is a numerical simulation program for non-isothermal flows of multicomponent, multiphase fluids in porous and fractured media. The modular architecture of TOUGH2 allows relatively easy extension to handle new fluid mixtures with new thermophysical properties. The appropriate equation-of-state module provides the thermophysical parameters as a function of a set of primary variables. EOS7 [Pruess, 1991b], for instance, describes the main effects of salinity on density and viscosity of the aqueous phase, and the dependence of gas solubility on brine concentration. As an extension of the EOS7 module, the new equation-of-state package EOS11 models the gelation process by specifying a Gel Time Curve and applying a Mixing Rule. The modeling approach is discussed in Section 2, and the thermophysical properties of the liquid phase containing grout are described in Section 3. The preparation of an input deck and application of the EOS11 module are demonstrated in Sections 4 and 5, respectively. 


\section{MODELING APPROACH}

\subsection{Process Description}

Injection of a water-based grout into an unsaturated porous medium leads to a system which consists of three separate phases, namely the solid grains, a non-condensable gas, and an aqueous phase of variable grout concentration. This three-phase description is correct, however, only if grout and water are completely miscible. Some grouts, such as polysiloxenes, are immiscible with water and therefore form a separate phase. The modeling of such systems is described elsewhere [Finsterle et al., 1994]. Even in the case of miscible grout solutions, such as colloidal silica, two stages have to be considered. At early times, the grout is dissolved in the aqueous solution. After some time, the gelling process is initiated, and the grout starts to form a separate phase, which turns into a non-Newtonian, visco-elastic fluid that eventually solidifies. The appearance of a new phase leads to changes of the physical and chemical properties as time proceeds. Contact angles and interfacial tensions vary with the chemical properties of the gel-water mixture, adsorption and filtration of gel clusters may occur. By the time the grout is completely gelled, a porous medium has been formed with a lower porosity, a new pore structure, reduced permeability, and probably different wettability characteristics.

Gelation kinetics not only depend on the composition of the grout, but also on the soil mineralogy and pore water chemistry. It has been shown, for example, that the gel time of colloidal silica increases with increasing $\mathrm{pH}$ and with decreasing ionic strength [Iler, 1979]. It is therefore affected by the pore water salinity, and by the multi-valent ions that desorb from clays and ion-exchange for mono-valent ions in the grout [Moridis et al., 1994].

Numerical models designed to simulate in situ gelation displacements for enhanced oil recovery have been presented in the literature [Scott et al., 1985; Hortes, 1986; Todd, 1990]. The main emphasis in these works is on the kinetic models of the gelation process. The most sophisticated simulator was developed by Todd [1990]. It combines transport equations for ten components with models of gelation kinetics, deposition, compaction and filtration of gel aggregates which leads to a reduction of porosity and increase of flow resistance. This model has been successful in qualitatively reproducing the data from one-dimensional laboratory gel displacement experiments performed by $\mathrm{McCool}$ [1988]. It requires, however, a large number of parameters which are difficult to determine. 
The work on the emplacement of subsurface barriers performed at the Lawrence Berkeley Laboratory [Moridis et al., 1994] focuses on the injection of grout materials into the unsaturated zone. In order to ensure a complete sealing of the pore space, it is crucial to control both the gelling process as well as the emplacement and flow of the gelling fluid in the unsaturated porous medium. Barrier fluids have been identified which are unaffected by the soil chemistry. This allows controlling the gel performance by appropriately designing the composition of the grout. Some barrier fluids, however, require a special pre-treatment of the soil [Moridis et al., 1994].

In our modeling approach, two major assumptions are made.

(1) The chemical process of gelation is not explicitly modeled. Instead, we calculate the viscosity of the aqueous phase as a function of grout concentration and time. The viscosity of pure grout as a function of time is measured in the laboratory and represented by a Gel Time Curve. Mixture viscosity varies with the concentration of gel in the aqueous phase, and is described by a Mixing Rule.

(2) Initially, the grout is treated as a miscible aqueous solution, therefore not forming a separate phase. After completion of the gelling process, we assume that the gel (which is a fluid of very high viscosity) solidifies instantaneously. By doing so, the porosity is reduced. The new porous medium thus has a lower permeability and different characteristic curves in the region affected by the grout. The transition of the grout from a highly viscous fluid to a solid part of the matrix is described by the Solidification Model.

Gel Time Curve, Mixing Rule, and Solidification Model are discussed in the following section. TOUGH2 is used to model non-isothermal subsurface flow of groundwater, air, and grout. A complete description of the physical processes, governing equations, program architecture, and user features is given in the TOUGH and TOUGH2 user's guides [Pruess, 1987,1991 a]. This report describes only the additional features that have been attached to the TOUGH2 code to simulate the flow of aqueous gelling liquids.

\subsection{Fluid Properties}

In the model, the pore space is occupied by two fluids: the gaseous phase, consisting of air and water vapor, and the liquid phase which is composed of water, grout, and dissolved 
air. The gaseous phase is treated as ideal, and additivity of vapor partial pressures is assumed. Dissolution of air in the liquid is represented by Henry's law. Thermophysical properties of liquid water and vapor are taken from steam table equations, as given by the International Formulation Committee [1967]. For grout-water mixtures it is assumed that the grout and water volumes are additive, which results in a mixture density of the liquid phase $\rho_{1}$ given by:

$$
\frac{1}{\rho_{1}}=\frac{1-X_{1}^{\text {gel }}}{\rho_{w}}+\frac{X_{1}^{\text {gel }}}{\rho_{\text {gel }}}
$$

where $\rho_{w}$ is water density, $\rho_{\text {gel }}$ is the density of the gel, and $X_{1}^{\text {gel }}$ is the mass fraction of gel in the liquid phase. We further assume that the expansivity and compressibility of the liquid grout are equal to the values for pure water at all temperatures and pressures. Thus the relative change of density as a function of temperature and pressure for grout is the same as for pure water:

$$
\frac{\rho_{\text {gel }}(\mathrm{P}, \mathrm{T})}{\rho_{\mathrm{gel}}\left(\mathrm{P}_{0}, \mathrm{~T}_{0}\right)}=\frac{\rho_{\mathrm{w}}(\mathrm{P}, \mathrm{T})}{\rho_{\mathrm{w}}\left(\mathrm{P}_{0}, \mathrm{~T}_{0}\right)}
$$

To calculate mixture density at arbitrary temperature, pressure, and grout concentration we use Eq. (2) for grout density in Eq. (1). The density of the reference grout at $T_{0}$ and $P_{0}$ can be specified by the user. By default, grout density will be assumed to be identical to the one of pure water.

As outlined in Section 2.1, the viscosity of the liquid phase depends on grout concentration and time. The increase of pure grout viscosity as a function of time is described by the Gel Time Curve, a parameterized function which can be fitted to laboratory data. Based on the measurements of Moridis et al. [1994] we suggest to use an exponential function of the form:

Gel Time Curve:

$$
\mu_{\mathrm{gel}}=\mathrm{a}_{1}+\mathrm{a}_{2} \cdot \exp \left(\mathrm{a}_{3} \cdot \mathrm{t}\right)
$$

where $t$ is time, and $a_{1}, a_{2}$, and $a_{3}$ are fitting parameters. User-provided Gel Time Curves can be programmed into subroutine GELLING. The structure of the subroutine is described in 
Appendix A. The relative change of gel viscosity as a function of temperature is assumed to be the same as for pure water:

$$
\frac{\mu_{\text {gel }}(T)}{\mu_{\text {gel }}\left(T_{0}\right)}=\frac{\mu_{w}(T)}{\mu_{w}\left(T_{0}\right)}
$$

The user may add a Gel Time Curve which is also a function of temperature.

After injection, the grout suspension will be diluted due to mixing with pore water. One of the following Mixing Rules can be applied to calculate the viscosity of the liquid phase, $\mu_{\mathrm{l}}$, as a function of gel concentration, $\mathrm{X}_{1}^{\mathrm{gel}}$, and time:

Linear Mixing Rule:

$$
\mu_{1}=X_{1}^{g e l} \cdot \mu_{\text {gel }}+\left(1-X_{1}^{g e l}\right) \cdot \mu_{w}
$$

Power-Law Mixing Rule: $\quad \mu_{1}=\left(\frac{X_{1}^{g e l}}{\mu_{g e l} b}+\frac{1-X_{1}^{g e l}}{\mu_{w}^{b}}\right)^{-1 / b}$

Todd [1990] suggests using a quarter-power mixing rule, i.e. $b=0.25$ in Eq. (5b).

Figure 1 shows the viscosity of the liquid phase as a function of time and gel concentration. The upper part of Figure 1 shows the viscosity measurements for a colloidal silica gel (symbols). The solid line is the fitted gel time curve (Eq. 3) which provides the viscosity as a function of time for pure gel $\left(\mathrm{X}_{1}^{\mathrm{gel}}=1\right.$ ). A linear mixing rule (Eq. 5a) has been applied and is visualized in the lower part of the Figure where viscosity (in centipoise) of the liquid phase is contoured as a function of gel concentration and time.

Recall that Gel Time Curve and Mixing Rule are only meaningful as long as the grout is completely miscible with water, and as long as the grout-water mixture behaves like a Newtonian fluid. 

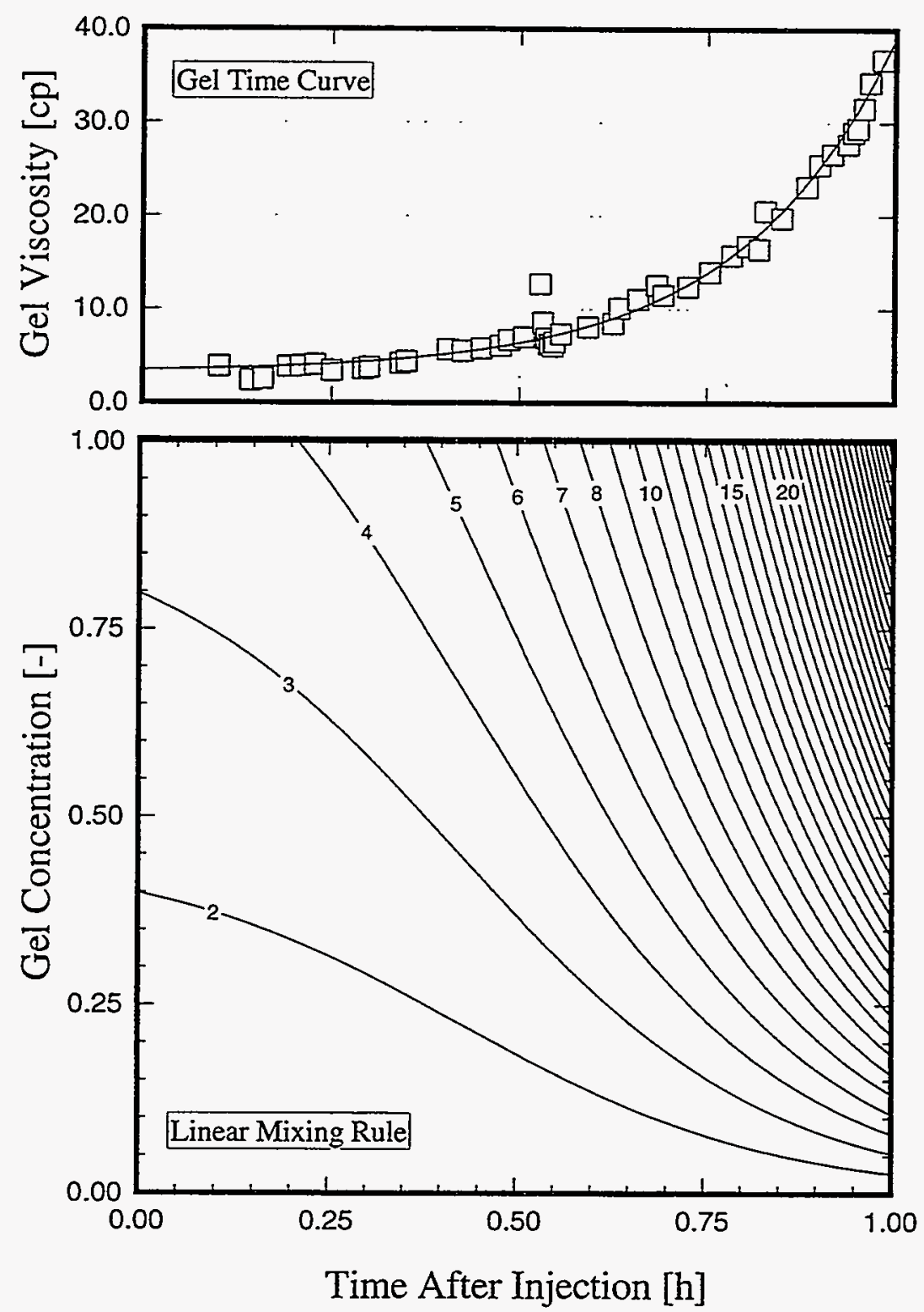

Figure 1: Viscosity [cp] of pure gel as a function of time (Gel Time Curve), and gel concentration (Mixing Rule); temperature is $20^{\circ} \mathrm{C}$. 


\subsection{Solidification Model}

As discussed in the previous section, the aqueous grout is modeled as a liquid, the viscosity of which increases with time as gelation proceeds. One should realize, however, that gelation in a porous medium is a far more complicated process. Crosslinking gels, for example, form aggregates when individual polymer chains become chemically crosslinked or physically entangled with other polymer chains. The size of these aggregates increases during the gelation process, and in the absence of shear stresses, a single large gel molecule is ultimately formed throughout the volume of gelling solution. If flow occurs, gel clusters may also be removed from the gelling solution by depth filtration and adsorption of gel aggregates to soil particles [Todd, 1990]. The fact that a new phase evolves, initially as a separate liquid phase, and eventually as a solid phase forming a part of the soil matrix, is not explicitly accounted for in our model. Nevertheless, the properties of the new porous medium after complete solidification of the grout can be described by making some assumptions about the new pore structure. The assumption of instant phase change may be a valid simplification because the transport velocity of highly viscous grout just prior to solidification is very low: Grout emplacement is controlled by the early time behavior of the system, when low viscosity grout is applied under high injection pressures.

Table 1 shows the major components in the gas, liquid, and solid phases, and how the Solidification Model has to be applied to perform the phase change in the regions affected by grout. Note that the gas phase also contains water vapor, and that small amounts of air are dissolved in the liquid phase according to Henry's law.

\begin{tabular}{|c|c|c|c|c|}
\hline \multicolumn{4}{|c|}{ Major Component in Phase } & Comment \\
\hline Air & Water & Grout & Soil & \\
\hline gas & \multicolumn{2}{|c|}{ liquid } & solid & grout and water are miscible \\
\hline gas & \multicolumn{2}{|c|}{ grout-water mixture solidifies } & apply solidification model \\
\hline gas & liquid & solid & new porous medium \\
\hline
\end{tabular}

Table 1: Concept and application of Solidification Model

The Solidification Model presented in the following paragraphs provides a new parameter set for the grouted soil. The parameters to be recalculated are porosity, 
permeability, relative permeability and capillary pressure functions, and initial liquid saturation. They are basically a function of the final grout content. Note that an initially homogeneous soil becomes heterogeneous with lower porosities and permeabilities in regions with a high final grout content. On the other hand, an initially heterogeneous soil may become more homogeneous on a scale smaller than the plume size, because highly permeable zones are preferentially clogged with grout compared to regions which are already relatively impermeable.

The Solidification Model is based on the assumption that all the liquid in the pore space eventually solidifies if the grout in the liquid phase exceeds a certain minimum concentration, $\mathrm{X}_{\min }$. We introduce a parameter $\mathrm{A}$ as follows:

$$
\begin{array}{cl}
A=1 & \text { for } X_{1}^{\text {gel }} \geq X_{\text {min }} \\
A=\frac{X_{1}^{\text {gel }}}{X_{\text {min }}} & \text { for } X_{1}^{\text {gel }}<X_{\text {min }}
\end{array}
$$

Setting $X_{\min }$ to 0.2 , for example, means that all the liquid with a gel concentration greater than 0.2 eventually solidifies. The fluid with lower gel concentrations solidifies incompletely.

The liquid saturation at the time solidification occurs is denoted by $S_{1}$. All soil characteristics and initial conditions referring to the new porous medium are denoted by a star $\left(^{*}\right)$. The porosity of the grouted sand is reduced by the amount of gel that solidified:

$$
\phi^{*}=\phi\left(1-\mathrm{A} \cdot \mathrm{S}_{\mathrm{I}}\right)
$$

The porosity reduction leads to a decrease of absolute permeability. The partial clogging of the pore space by grout is conceptually similar to the permeability reduction due to phase interferences in a multiphase flow system. The Permeability Reduction Model (PRM) is a function that provides the permeability depending on the solidified grout saturation:

$$
\mathrm{k}^{*}=\mathrm{k} \cdot \mathrm{PRM}
$$

Since the grout-water mixture is the wetting fluid, one might take the relative permeability function of the non-wetting phase to calculate the absolute permeability of the grouted soil: 


$$
\begin{gathered}
-9- \\
\left.\mathrm{PRM}=\mathrm{k}_{\mathrm{rg}}\right)_{(\mathrm{Sg}=1-\mathrm{A} \cdot \mathrm{Sl})}
\end{gathered}
$$

The permeability reduction might in fact be stronger, because not only are the small pores sealed by the wetting grout, but continuous gel adsorption at the pore walls reduces the diameter of the remaining larger pores. Todd [1990] proposes the following Permeability Reduction Model:

$$
\operatorname{PRM}=\left(1-\mathrm{A} \cdot \mathrm{S}_{1}\right)^{\mathrm{m}}
$$

The Blake-Kozeny-Carman model reads [Oldenburg and Spera, 1992]:

$$
\operatorname{PRM}=\frac{\left(1-A \cdot S_{1}\right)^{3}}{\left(A \cdot S_{1}\right)^{2}}
$$

Due to the reduced pore sizes, the capillary pressure of the grouted sand is expected to be more negative for a given water content. We apply Leverett's model to calculate the capillary pressure of the medium with reduced permeability. Furthermore, the change of the wetting characteristics (i.e. contact angle $\alpha$ ) has to be accounted for, and the saturations have to be scaled to the new porosity and liquid saturation of the sand:

$$
\mathrm{p}_{c}{ }^{*}=\mathrm{p}_{\mathrm{c}}\left(\mathrm{S}_{1, \mathrm{ori}}\right) \cdot \sqrt{\frac{\mathrm{k}}{\mathrm{k}^{*}}} \cdot\left[1-\mathrm{A}\left(1-\frac{\cos \alpha^{*}}{\cos \alpha}\right)\right]
$$

where

$$
S_{1, \text { ori }}=A \cdot S_{1}+S_{1}^{*} \cdot \frac{\phi^{*}}{\phi}=A \cdot S_{1}+S_{1}^{*} \cdot\left(1-A \cdot S_{1}\right)
$$

Given a certain liquid saturation $\mathrm{S}_{1}{ }^{*}$ of the grouted sand, Eq. (11) gives the corresponding original liquid saturation $S_{l, o r i}$ which yields the equivalent water content. The capillary pressure is then obtained and enhanced by applying Leverett's scaling model using Eq. (10). This simplified approach assumes that the pore size distribution is shifted but that its shape is not changed. Consequently, the exponents in the commonly used characteristic curves by Brooks-Corey (Eq. 14) and van Genuchten (Eq. 16) are not modified, either.

The liquid saturation after solidification, $\mathrm{S}_{10}{ }^{*}$, is the volume of the ungelled pore fluid divided by the new pore volume from Eq. (7):

$$
S_{10}^{*}=\frac{S_{1} \cdot(1-A)}{1-A \cdot S_{1}}
$$


Finally, the gel concentration in the unsolidified fluid is recalculated:

$$
\mathrm{X}_{\mathrm{l}}^{\mathrm{gel} *}=(1-\mathrm{A}) \cdot \mathrm{X}_{1}^{\mathrm{gel}}
$$

Note that if new Gel Time Curves are applied (e.g. for modeling a secondary grout injection), the residual gel content from a previous injection behaves like newly injected grout. This usually small amount of grout resides mainly at the disperse interface between the grouted region and the zone which is not affected by gelation.

The Solidification Model has to be applied to each grid block of the discretized flow region to provide initial conditions and soil properties for subsequent simulations.

\section{USING THE Gelation MODUle EOS11}

\subsection{Installation}

EOS11 (file eos $11 . f$ ) is fully compatible with ITOUGH2, Version 2.2 [Finsterle, 1993], and can be compiled and linked in the same manner as the other EOS modules available. Note that MNK has to be set equal to 3, and MNEQ to 4 in the main program. If using the original TOUGH2 code [Pruess, 1987, 1991], new versions of the main program as well as subroutines INPUT, RFILE, MULTI, WRIFI, RELP, and PCAP are needed. This is necessary because after solidification, each grid block has its own absolute permeability value and coefficients for the capillary pressure function. Furthermore, the Solidification Model has to be applied before writing all primary variables onto file SAVE for restarting.

The modified subroutines are concatenated together as file $t 2 \mathrm{gel} . f$ which has to be compiled and linked in front of the standard TOUGH2 subroutines with the eos $11 . f$ equation-of-state module. If the EOS11 module is used together with the original TOUGH2 code (without file $t 2$ gel.f), grout injection and gelation can still be simulated, but no application of the Solidification Model is possible.

The gelation module can also be used together with the ITOUGH2 code which allows both solving the forward problem and performing multiple optimization runs for parameter 
estimation. No 2 gel.f file is needed in this case because all necessary modifications are already part of the standard ITOUGH2 code.

A summary of EOS11 specifications and options is printed upon program execution, and is reproduced here in Figure 2.

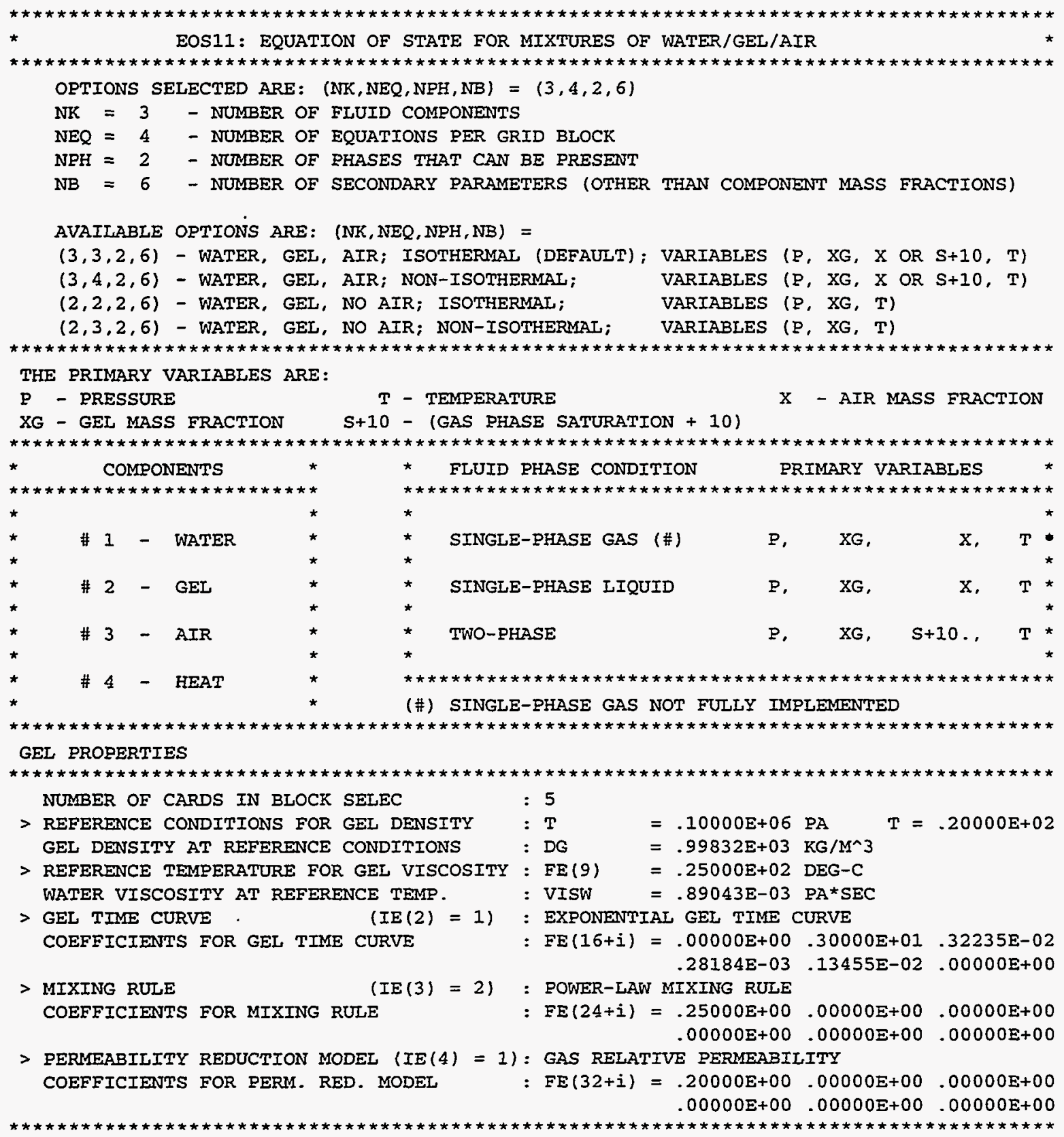

Figure 2: Summary of EOS11 specifications and options 


\subsection{Input Formats}

Injection of grout at a given rate can be specified by setting character variable TYPE in block GENER.1 equal to 'COM2'. The coefficients for the Gel Time Curve, the Mixing Rule, and the Solidification Model are specified in data block SELEC as follows:

SELEC Keyword to introduce parameters for gelation model

SELEC.1 Format (4I5)

$\operatorname{IE}(1), \operatorname{IE}(2), \operatorname{IE}(3), \operatorname{IE}(4)$

$\operatorname{IE}(1) \quad$ Set equal to 4 (or 5), to read four (or five) additional data records.

IE(2) Selects type of Gel Time Curve.

0 : No gelation

1: Exponential Gel Time Curve (Eq. 3)

Use higher numbers for your own Gel Time Curve programmed into subroutine GELLING (see Appendix A).

IE(3) Selects type of Mixing Rule.

1: Linear Mixing Rule (Eq. 5a)

2: Power-Law Mixing Rule (Eq. 5b)

Use higher numbers for your own Mixing Rules programmed into subroutine GELLING (see Appendix A).

IE(4) Selects type of Permeability Reduction Model.

0: Solidification Model not applied

1: Use gas relative permeability function (Eq. 9a)

2: Use Power-Law Permeability Reduction Model (Eq. 9b)

SELEC.2 Format (3E10.4)

$\mathrm{FE}(1)-\mathrm{FE}(3)$

$P_{0}, T_{0}, \rho_{0}$

$\mathrm{P}_{0}$ : $\quad$ Reference pressure for grout density, in $\mathrm{Pa}$

$\mathrm{T}_{0}$ : $\quad$ Reference temperature for grout density, in ${ }^{\circ} \mathrm{C}$

$\rho_{0}$ : $\quad$ Grout density at $\left(\mathrm{P}_{0}, \mathrm{~T}_{0}\right)$, in $\mathrm{kg} / \mathrm{m}^{3}$

For $P_{0}, T_{0}, \rho_{0}$ equal to zero or blank, grout will be assumed to have the same density as pure water. 
SELEC.3 Format (E10.4)

$\mathrm{FE}(9)$

$\mathrm{T}_{0}$

$\mathrm{T}_{0}$ : $\quad$ Reference temperature for grout viscosity (Eq. 4 ), in ${ }^{\circ} \mathrm{C}$

SELEC.4 Format (7E10.4)

$\mathrm{FE}(17)-\mathrm{FE}(23)$

Parameters for Gel Time Curve.

For exponential Gel Time Curves (Eq. 3, $\operatorname{IE}(2)=1$ ), the parameters are as follows:

FE(17): Time at which gelation starts, in sec

FE(18): Scaling factor for Gel Time Curve (default $=1.0$ )

FE(19): Coefficient $a_{1}$ of exponential Gel Time Curve (Eq. 3)

FE(20): Coefficient $a_{2}$ of exponential Gel Time Curve (Eq. 3)

FE(21): Coefficient a3 of exponential Gel Time Curve (Eq. 3)

$\mathrm{FE}(22)$ : Maximum viscosity, in Pa.s (default $=\infty$ )

$\mathrm{FE}(23)$ : Starting time for secondary grout injection, in sec (optional)

SELEC.5 Format (E10.4)

$\mathrm{FE}(25)$

Parameters for Mixing Rule.

For the Linear Mixing Rule (Eq. 5a, $\mathbb{E}(3)=1$ ), no coefficients are required.

For the Power-Law Mixing Rule (Eq. $5 b, \operatorname{IE}(3)=2)$, the parameter is:

$\mathrm{FE}(25)$ : Exponent $b$ in Eq. $5 \mathrm{~b}$ (default $=0.25$ )

SELEC.6 Format (3E10.4)

$\mathrm{FE}(33)-\mathrm{FE}(35)$

Parameters for Permeability Reduction Model.

FE(33): Minimum gel concentration for complete solidification ( $X_{\min }$ in Eq. 6)

For the Power-Law Permeability Reduction Model (Eq. 9b, $\operatorname{IE}(4)=2$ ), the parameter is:

FE(34): Exponent $m$ in Eq. (9b) (default $=3.0$ )

FE(35): Wettability changing factor $\left(\cos \alpha^{*} / \cos \alpha\right)$, Eq. (10) (default $\left.=1.0\right)$

At the end of a grout injection simulation, the Solidification Model can be applied to obtain the soil properties and the initial conditions of the grouted soil. If this new porous medium is used in subsequent simulation runs (for example to model a secondary grout 
injection or for performance assessment studies), highly heterogeneous soil characteristics have to be provided. Five variables are added to block INCON on file SAVE as follows:

INCON Keyword to introduce initial conditions.

INCON.1 Format (A3,12,215,E15.9,5E10.4)

EL,NE,NSEQ,NADD,PORX,(USERX(I),I=1,5)

The parameters EL, NE, NSEQ, NADD, and PORX are the same as in the standard TOUGH2 code. Subsequently, up to five user specified parameters can be read for each grid block. These are:

USERX(1): Absolute permeability after solidification of the gel

USERX (2): Capillary strength $=$ Air entry pressure ( $p_{e}$ in Eq. 14, or $1 / \alpha$ in Eq. 16) times Leverett's scaling factor (see Eq. 10)

USERX(3): Liquid saturation $S_{1, \text { sol }}$ at the time solidification occurred USERX(4): Grout concentration $X_{1}^{\text {gel }}$ at the time solidification occurred USERX(5): Parameter A (see Eq. 6)

INCON.2 Format (4E20.14)

$\mathrm{X} 1, \mathrm{X} 2, \mathrm{X} 3, \mathrm{X} 4$

Set of primary variables (as in TOUGH2, see also Figure 2)

The parameters stored in array USERX are now available for the calculation of elementspecific flow characteristics. The permeability stored in USERX(1), if not zero, overwrites the value provided through block ROCKS. The values stored in USERX(2) through USERX(5) are used in conjunction with specially programmed versions of Brooks-Corey's and van Genuchten's model. The equations for the Brooks-Corey model (BC) are [Brooks and Corey, 1964]:

$$
\begin{gathered}
\mathrm{p}_{\mathrm{c}}=\mathrm{p}_{\mathrm{e}} \cdot \mathrm{S}_{\mathrm{e}}^{-1 / \lambda} \\
\mathrm{k}_{\mathrm{rl}}=\mathrm{S}_{\mathrm{e}}^{\frac{2+3 \lambda}{\lambda}} \\
\mathrm{k}_{\mathrm{rg}}=\left(1-\mathrm{S}_{\mathrm{e}}\right)^{2} \cdot\left(1-\mathrm{S}_{\mathrm{e}}^{\frac{2+\lambda}{\lambda}}\right)
\end{gathered}
$$

with the effective liquid saturation

$$
S_{e}=\frac{S_{l}-S_{l r}}{1-S_{l r}} \quad\left(S_{l r}<S_{1}<1\right)
$$


and for van Genuchten's model (VG) in the notation of Luckner et al. [1989]:

$$
\begin{gathered}
\mathrm{P}_{\mathrm{c}}=-\frac{1}{\alpha}\left(\mathrm{S}_{\mathrm{e}}^{-1 / \mathrm{m}}-1\right)^{1 / \mathrm{n}} \\
\mathrm{k}_{\mathrm{rl}}=\mathrm{S}_{\mathrm{e}}^{1 / 2} \cdot\left[1-\left(1-\mathrm{S}_{\mathrm{e}}^{1 / \mathrm{m}}\right)^{\mathrm{m}}\right]^{2} \\
\mathrm{k}_{\mathrm{rg}}=\left(1-\mathrm{S}_{\mathrm{e}}\right)^{1 / 3} \cdot\left[1-\mathrm{S}_{\mathrm{e}}^{1 / \mathrm{m}}\right]^{2 \mathrm{~m}}
\end{gathered}
$$

After solidification, each grid block has its own capillary strength according to Eqs. (10) and (11). Either BC or the VG model should be used for a continuation run after solidification. The parameters to be specified in block RPCAP.1 and RPCAP.2 or ROCKS1.2 and ROCKS1.3 are:

RPCAP.1 or ROCKS1.2 Format (I5,5X,2E10.4)

$$
\text { IRP,RP(1),RP(2) }
$$

IRP: $\quad$ Set equal to 10 for choosing BC's and 11 for VG's model

$\mathrm{RP}(1)$ : $\quad$ Residual liquid saturation $\left(\mathrm{S}_{\mathrm{lr}}\right.$ in Eq. 15)

RPCAP.2 or ROCKS1.3 Format $(15,5 \mathrm{X}, 4 \mathrm{E} 10.4)$

$\mathrm{ICP},(\mathrm{CP}(\mathrm{I}), \mathrm{I}=1,4)$

ICP: $\quad$ Set equal to 10 for choosing BC's and 11 for VG's model

$\mathrm{CP}(1)$ : $\quad$ Pore size distribution index $\lambda(\mathrm{BC})$; parameter $\mathrm{n}(\mathrm{VG})$

$\mathrm{CP}(2)$ : $\quad$ Air entry pressure $\mathrm{p}_{\mathrm{e}}(\mathrm{BC})$; parameter $1 / \alpha(\mathrm{VG})$; overwritten by nonzero variable USERX(2).

$\mathrm{CP}(3)$ : $\quad$ Maximum capillary pressure (default $=\infty$ )

$\mathrm{CP}(4)$ : $\quad$ Parameter $m$ (VG only, default: $m=1-1 / \mathrm{n}$ )

$\mathrm{CP}(7)$ : Give negative number if Solidification Model shall not be applied to corresponding rock type (e.g. cleaned boreholes)

The capillary pressure functions $\mathrm{ICP}=10$ and $\mathrm{ICP}=11$ can be used in conjunction with the relative permeability functions IRP $=3,4,6,8,10$, and 11 . In order to have a consistent set of characteristic curves in the sense of Burdine [1953] and Mualem [1978], it is suggested to use IRP $=\mathrm{ICP}=10$, and $\mathrm{IRP}=\mathrm{ICP}=11$ only. 


\subsection{Application of Solidification Model}

Recall that the Solidification Model should only be applied if the saturation distribution in the grouted area is not changing significantly. This state is usually achieved shortly after grout injection has been completed because the viscosity of the gel is supposed to increase quickly soon after emplacement. Immobilization of the grout plume is, therefore, also a function of the Gel Time Curve.

There are three ways of applying the Solidification Model: (1) automatically at the end of a simulation run, (2) using a postprocessor, and (3) at certain, user-specified times during the simulation.

(1) If parameter IE(4) in block SELEC.1 is not zero, the Solidification Model is automatically applied at the end of the simulation run. The state of the system is printed twice in the TOUGH2 output file, prior to solidification, and after solidification. The new initial conditions and the changed soil characteristics are written onto file SAVE which can be used for subsequent modeling studies of the grouted soil. Note that the primary variable No. 3 holds the saturation referring to the new porous medium with reduced porosity.

(2) If parameter IE(4) in block SELEC.1 is zero, the Solidification Model is not applied. This means that the content of file SAVE represents the state of the system at the end of the simulation, the pore space being filled with high viscosity gel. This information can be taken as input to a postprocessor, which produces an INCON file with the new initial conditions and characteristics of the soil after the gel has solidified. The FORTRAN source code of this postprocessor is listed in Appendix B. If the name of the executable is solimod.out, for example, the Solidification Model can be run using the following command: solimod. out < SAVE > INCON.

(3) The third option is only available if EOS11 is run using ITOUGH2. ITOUGH2 allows changing initial and boundary conditions at any point in time during a simulation. This feature can be used to apply the Solidification Model and start a continuation run without interruption. The following command sequence may be used in the ITOUGH2 input file to stop grout injection in borehole 1, apply the Solidification Model, and start injection in a second borehole at a constant pressure of 2 bar. The input reads as follows: 
Provide keyword > RESTART TIME: 1 and the time at which the Solidification Model shall be applied. Change initial conditions in grid block BOR_1 and BOR_2. The integer after the element code name indicates which primary variable is modified; a zero has to be given if the volume of the grid block is changed.

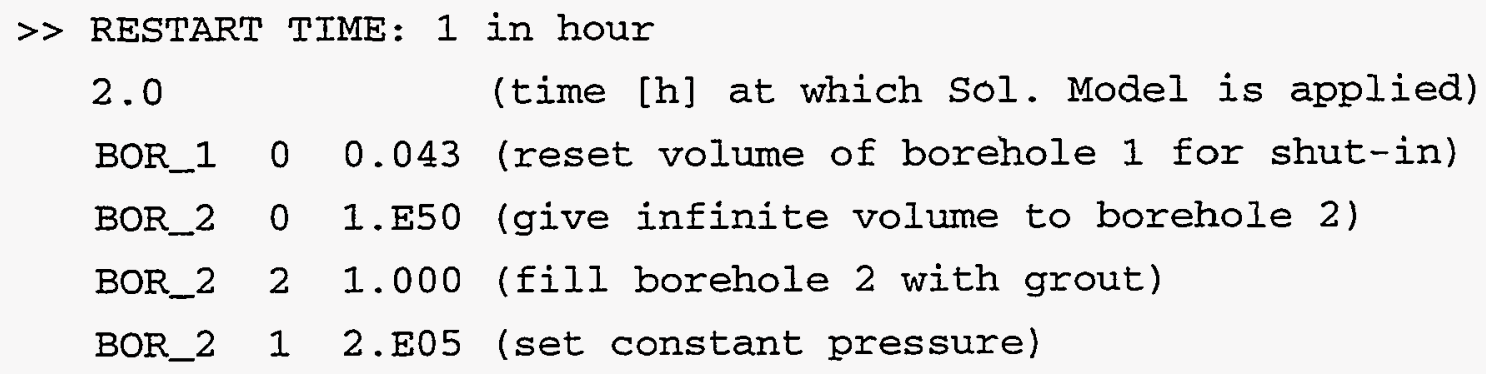

Note that if the grout used for the secondary injection has a different Gel Time Curve, this has to be programmed into subroutine GELLING. If the same Gel Time Curve applies, the starting time of the secondary injection has to be provided through variable $\mathrm{FE}(23)$ ( see Section 3.2).

As another example, one might apply the Solidification Model repeatedly in regular time intervals without changing boundary conditions. The command may be the following:

> RESTART AT: 3 EQUALLY SPACED TIMES BETWEEN $3600.0 \quad 7200.0$ SECONDS

The Solidification Model is coded in subroutine WRIFI, file $t 2$ gel.f. If using ITOUGH2, modifications of the standard Solidification Model have to be programmed into subroutine GETINCON, file it2MAIN.f. 


\section{SAMPLE PROBLEMS}

\subsection{Sample 1: Multiple Grout Injections Into Unsaturated Sand}

This sample problem considers multiple grout injections into initially dry sand. The simulation reflects the test sequence of a possible laboratory experiment. A small tank $(30 \times 30 \times 10 \mathrm{~cm})$ is filled with sand. Three horizontal holes are drilled as shown in Figure 3 . The two lower wells are used for a primary grout injection. The third borehole centered above is used for a secondary grout injection after the first plume has solidified. The top of the sandbox is open to atmospheric conditions.

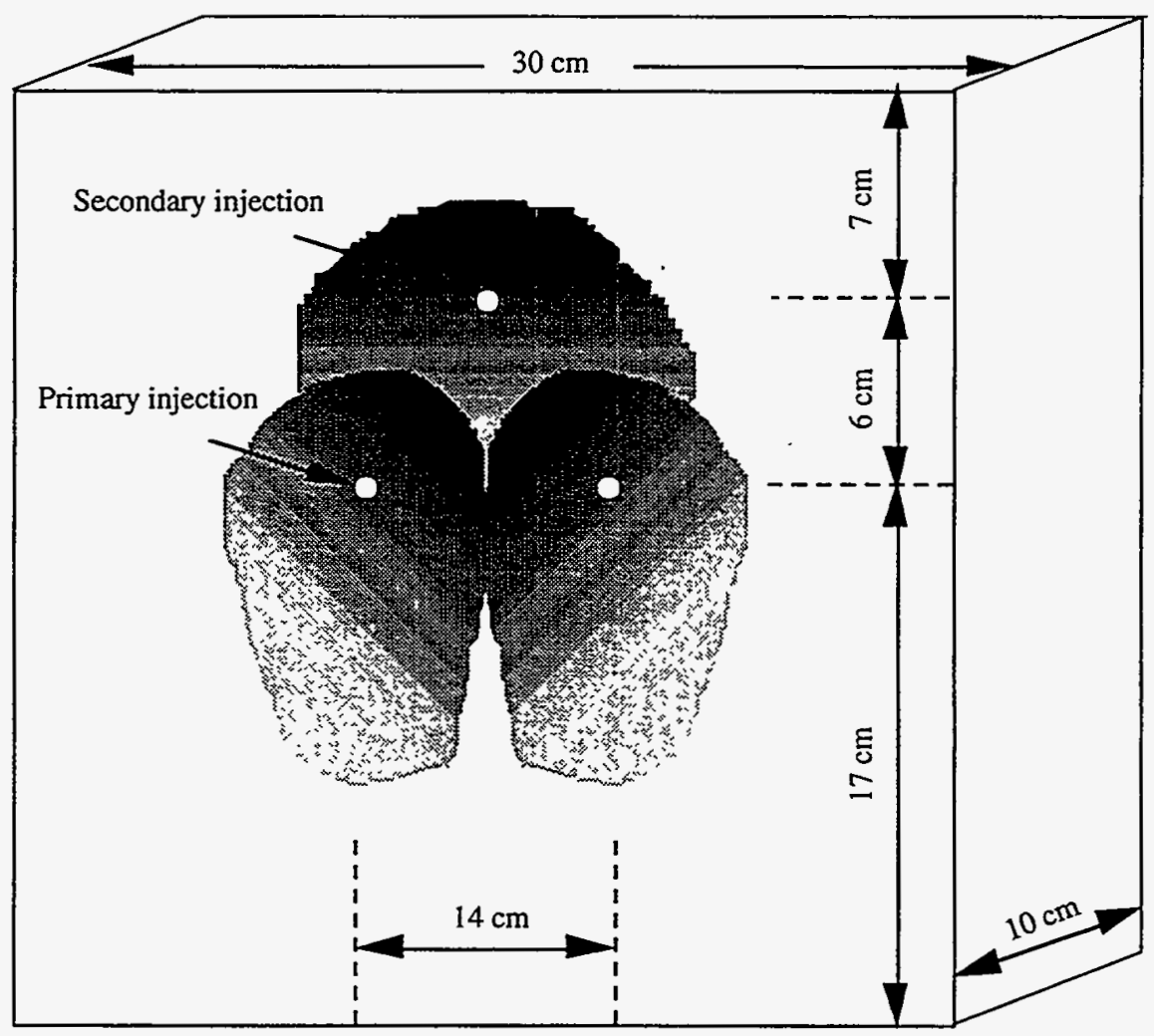

Figure 3: Schematic of sandbox and injected grout for sample problem No. 1

The characteristics of the sand are similar to those of Oklahoma \#1 sand. The saturated permeability is $8.0 \mathrm{E}-12 \mathrm{~m}^{2}$. Brooks-Corey's model is used to describe capillary pressure and relative permeability as a function of liquid saturation. We simulate the injection of colloidal silica grout. The gel time curve is the one shown in Figure 1 , and a quarter-power $(m=0.25)$ mixing rule is chosen. The chemical behavior of colloidal silica grouts is influenced by the presence of multi-valent ions in the soil and soil-water which tend to accelerate the gelation 
process. In order to ensure a controllable gel time, the ions preferentially adsorbed at the clay particles have to be removed by flushing the soil with water or, more effectively, by a saline solution. Recall that the chemical reactions are not explicitly modeled. Nevertheless, we simulate preflushing as a 30-minute water injection period to obtain the correct initial saturation distribution for the subsequent grout emplacement. Grout is injected at a constant rate for 10 minutes and allowed to redistribute for a period of 4.5 hours. The Solidification Model is applied, reducing porosity and permeability in the region affected by the grout plume. Furthermore, the capillary strength is increased. Subsequently, the secondary grout plume is injected and allowed to cure. The Solidification Model is again applied after 10 hours to obtain the final characteristics of the grouted sand.

The corresponding TOUGH2 input file is shown in Figure 4. An ITOUGH2 input file (Figure 5) is used to apply the Solidification Model at $\mathrm{t}=5$ hours without interrupting the simulation (see Section 3.3). Since no inversion is performed, the user does not need to specify TOUGH2 input parameters to be estimated nor observations. An appropriately extended ITOUGH2 input file could be used to determine, for example, parameters of the Solidification Model by fitting the model to measured saturation or pressure data observed during the secondary grout injection.

Figure 6 shows the saturation distribution after preflushing the sand with water at a constant rate of $12 \mathrm{ml} / \mathrm{min}$ in each well for 30 minutes. Subsequently, grout is injected for 10 minutes at a constant rate of $30 \mathrm{ml} / \mathrm{min}$ in the two lower wells. Figures 7 and 8 show the grout content immediately after injection and at $t=5$ hours, respectively. Grout content is defined as the product of grout concentration and liquid saturation. In Figure 7, grout content around the injection wells is close to 1 . The grout, however, becomes more disperse with time due to the spreading of the plume driven by capillary forces. The plume also slumps downward under gravity, but eventually becomes immobile due to the increase of gel viscosity. The maximum grout content in Figure 8 is only slightly above 0.6 . The porosity and permeability field after solidification is depicted in Figures 9 and 10, respectively. Note that the location of the maximum porosity and permeability reduction does not coincide with the location of the maximum grout content shown in Figure 8. Grout concentration in the lower part of the plume is lower than in the central part, but high enough to cause complete solidification of the gel-water mixture. Since liquid saturation is higher near the bottom of the sandbox, this region exhibits the largest porosity reduction. The permeability in this zone is reduced by more than two orders of magnitude. 
Sample Problem 1: Multiple Grout Injections Into Unsaturated Sand

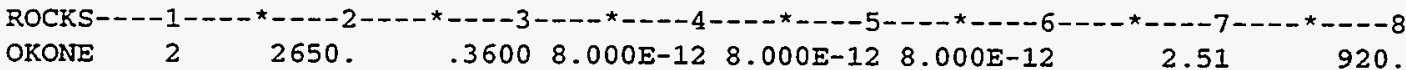

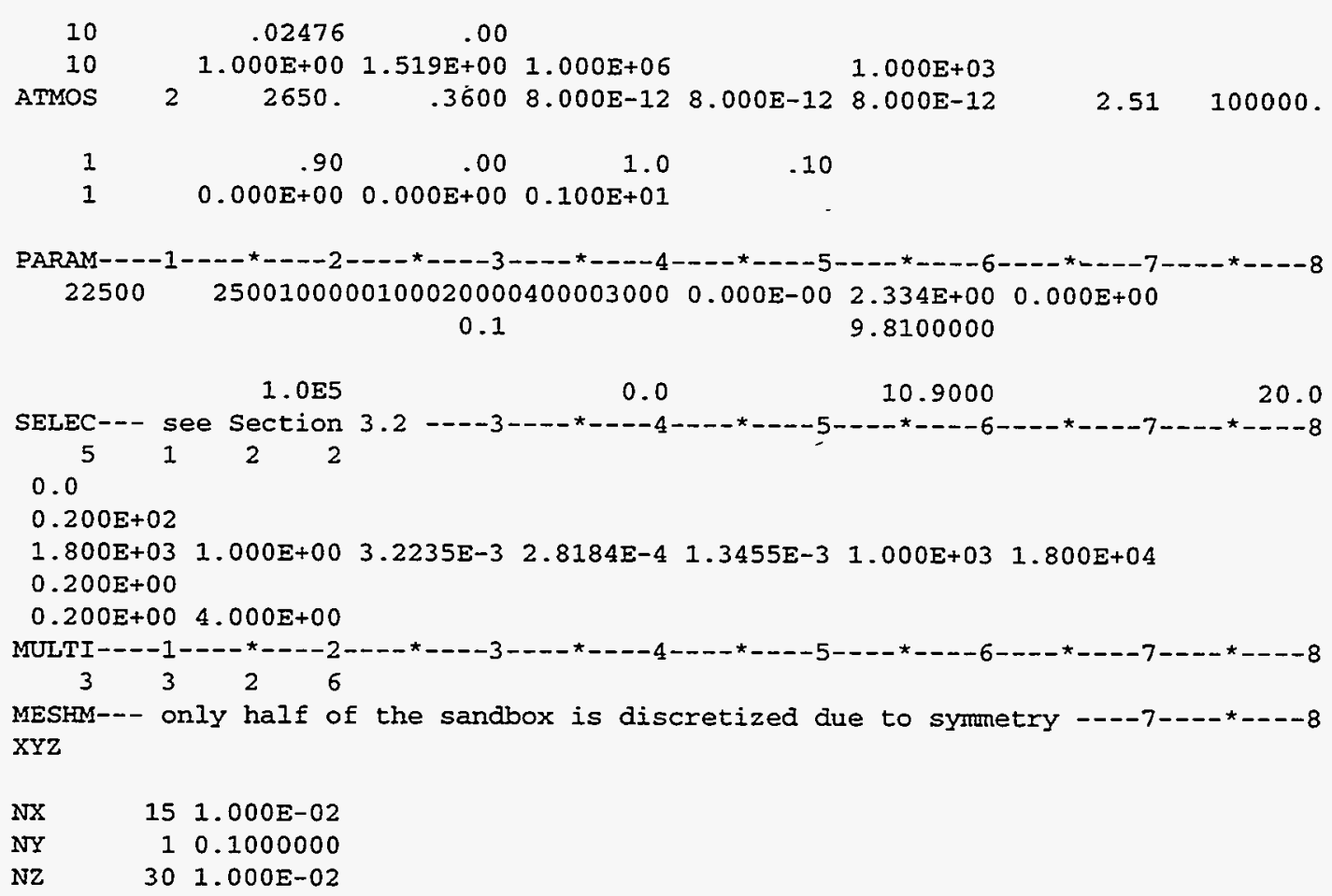

Figure 4: TOUGH2 input file for sample problem 1. 


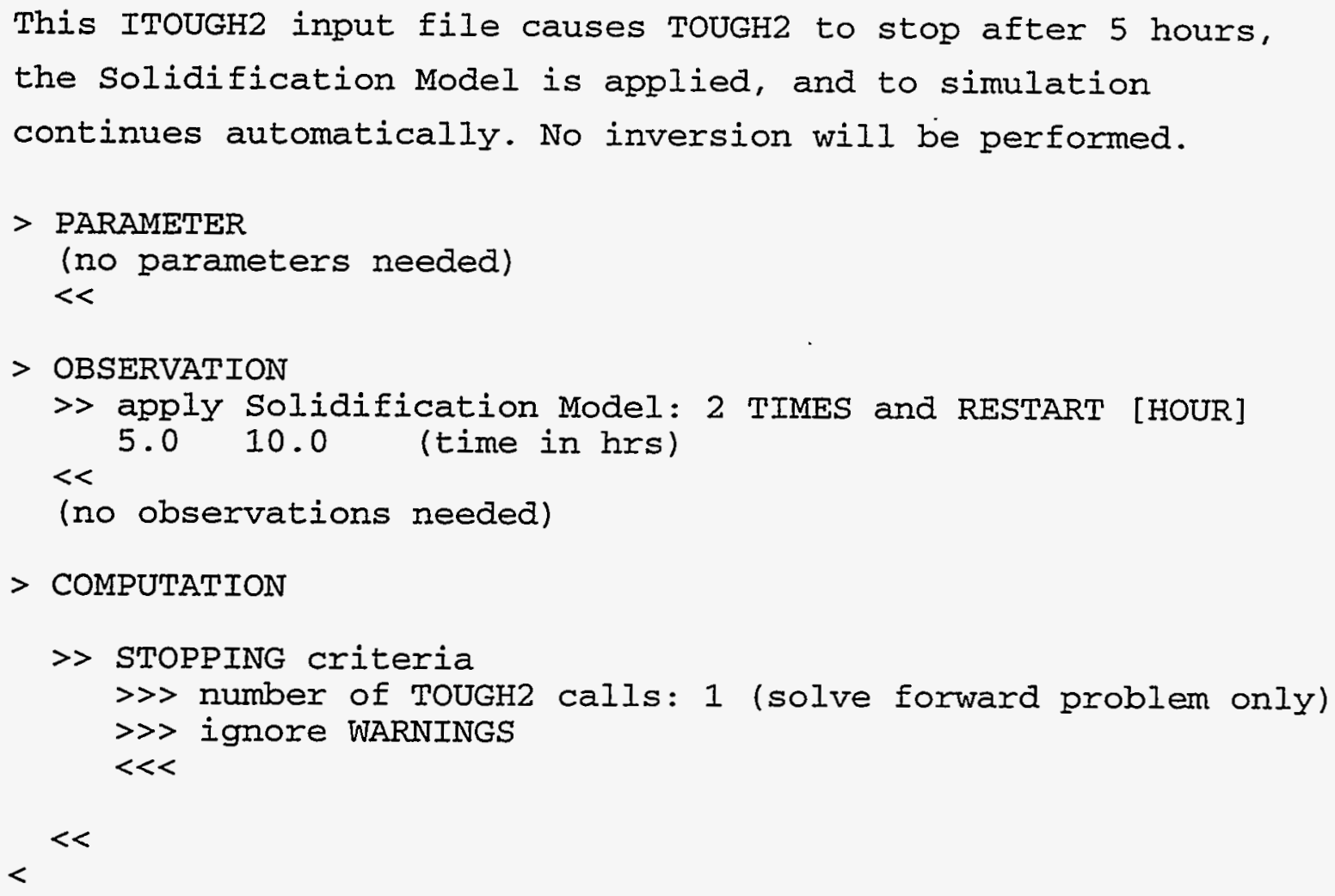

Figure 5: ITOUGH2 input file for sample problem 1.

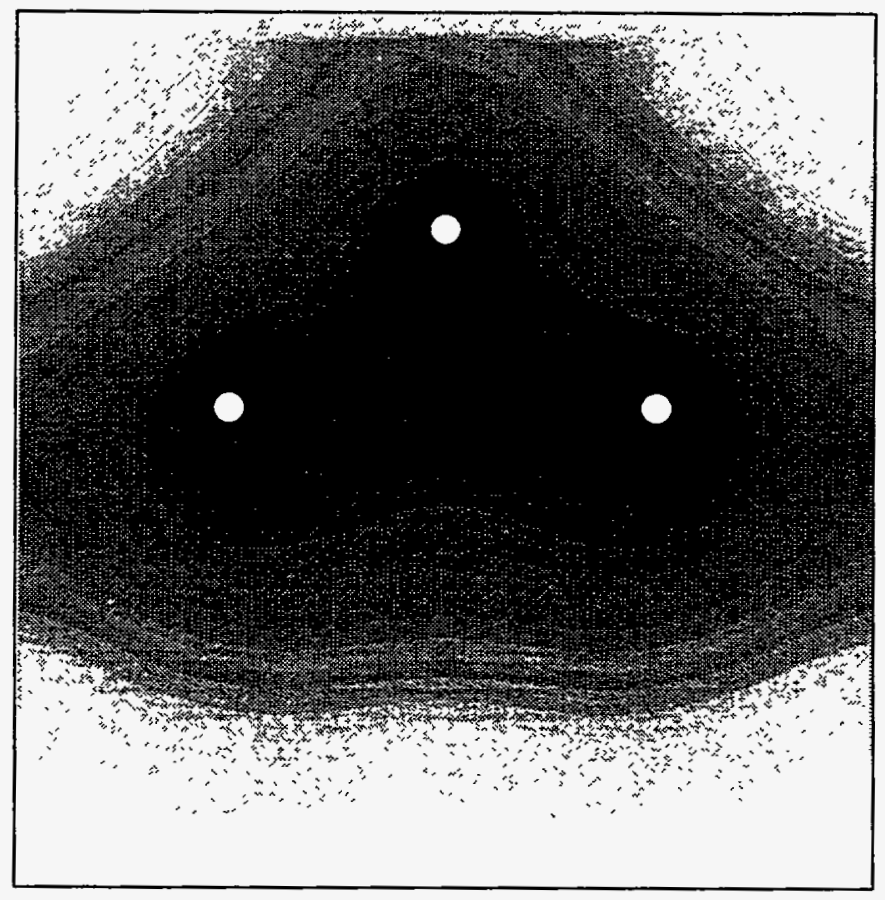

Figure 6: Sample problem 1: Liquid saturation after preflushing 


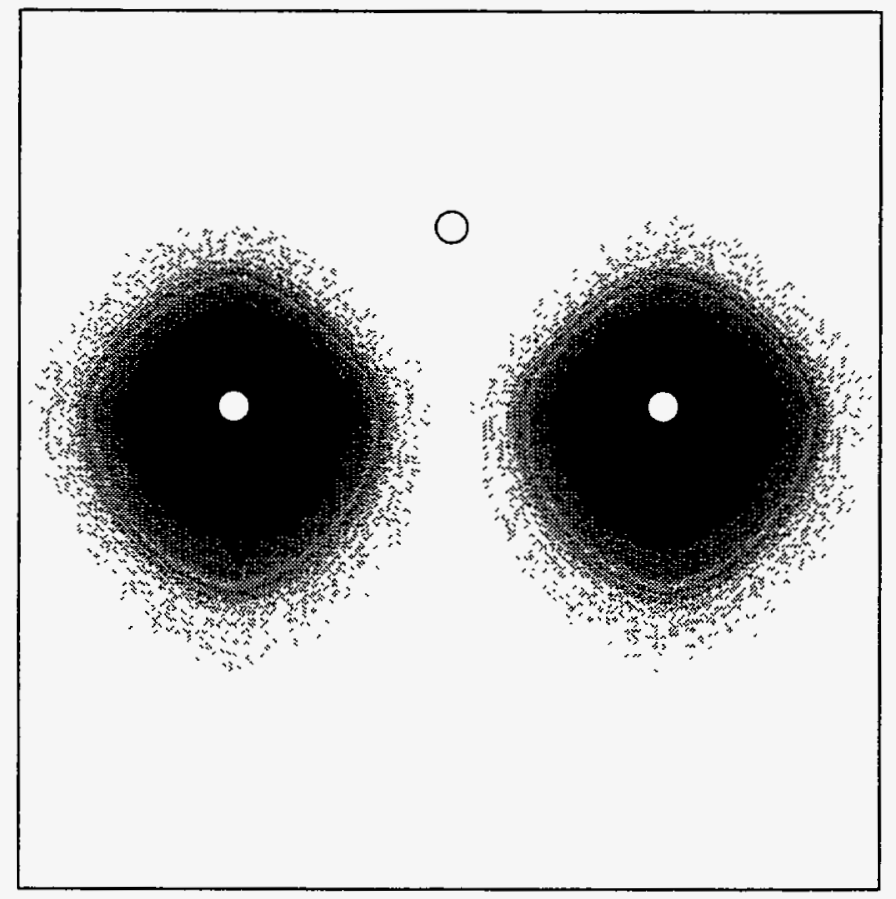

Figure 7: Sample problem 1: Grout content immediately after primary injection

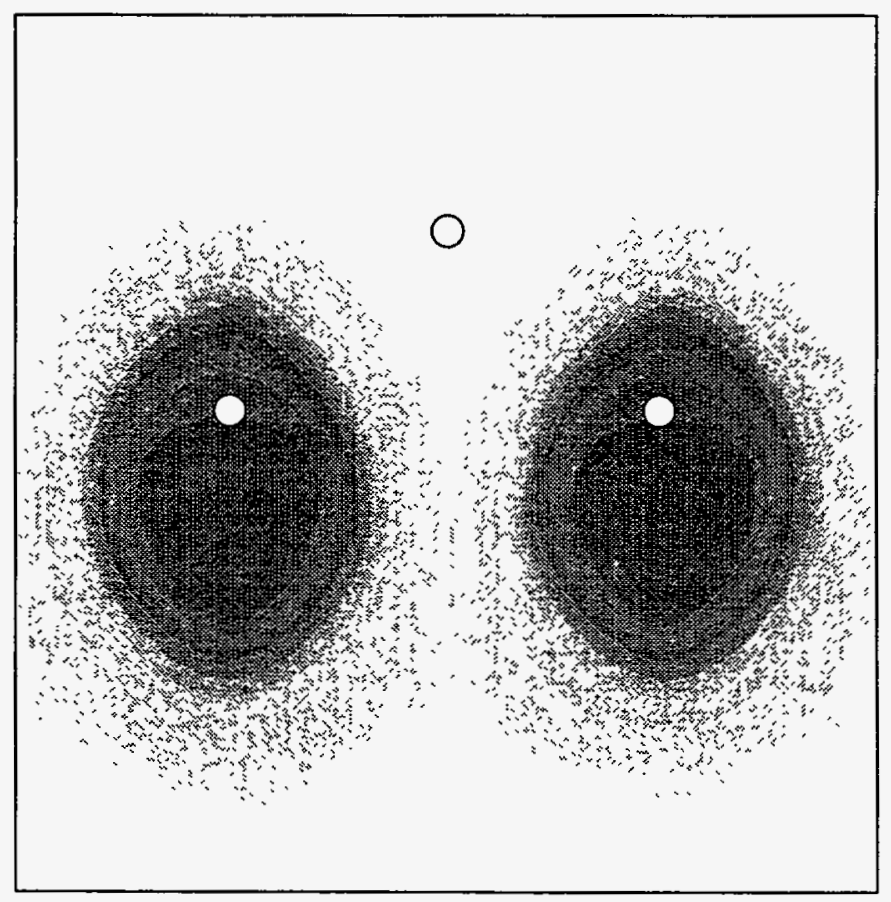

Figure 8: Sample problem 1: Grout content at $\mathrm{t}=5 \mathrm{hrs}$ 


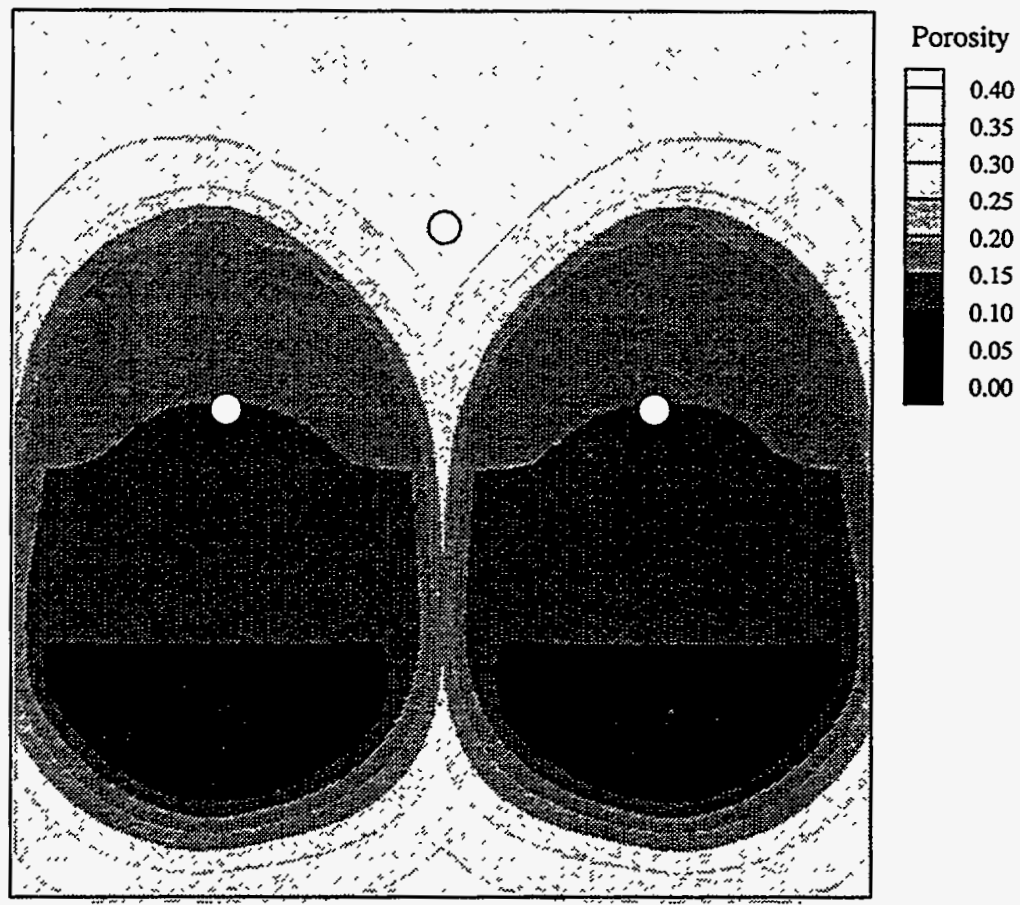

Figure 9: Sample problem 1: Porosity distribution after furst solidification

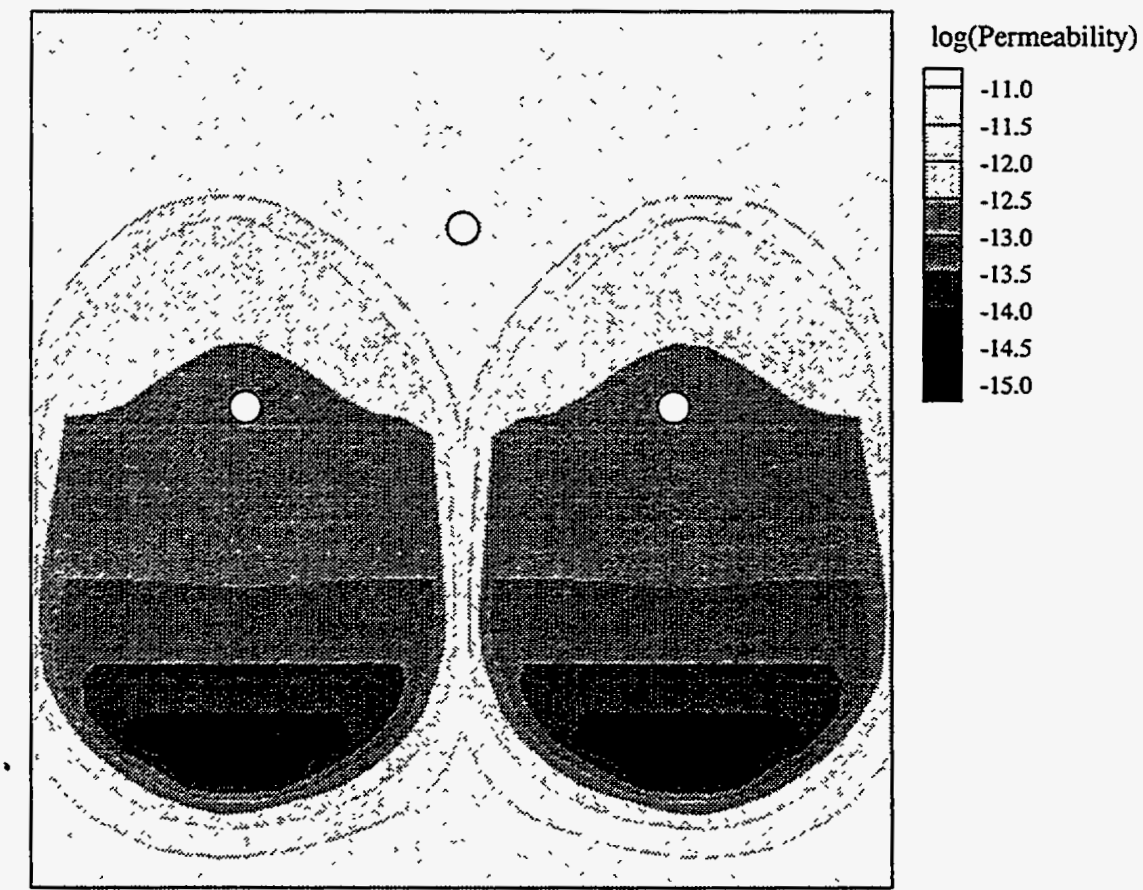

Figure 10: Sample problem 1: Log-Permeability distribution after first solidification 
A secondary injection of grout is performed from the center borehole at a constant rate of $30 \mathrm{ml} / \mathrm{min}$. The behavior of this secondary plume is governed by the heterogeneity of the grouted sand. The permeability field causes the plume to be diverted horizontally. At the same time, grout preferentially flows downward along the center line between the two primary plumes. This is qualitatively shown in Figure 11, where the grout content is visualized at the end of the injection period. With time, however, the secondary grout plume is sucked into the top part of the primary plumes due to the increased capillary strength. The grout content at $\mathrm{t}=10$ hours is shown in Figure 12. The Solidification Model is applied, and the final porosity and permeability field is plotted in Figures 13 and 14, respectively. The results of these simulations reveal the effect of multiple grout injections on soil permeability. The secondary injection leads to much stronger permeability reductions (permeability in the black area in Figure 14 is reduced by at least four and up to seven orders of magnitude compared to the initial permeability of $8.0 \mathrm{E}-12 \mathrm{~m}^{2}$ ). This effect is mainly due to the fact that the spreading of the secondary plume in the heterogeneous, low permeability sand is decreased, leading to higher grout contents at the time solidification occurs.

Figures 15 and 16 show an excerpt of the output file at $t=10$ hours prior to and after application of the Solidification Model, respectively. Note the decrease of porosity, permeability, and liquid saturation in the grid blocks with significant grout concentration in the liquid phase.

It is important to realize that the results shown for this sample problem strongly depend on the formulation and the parameters of the Solidification Model. Not only is the final permeability field directly related to the Permeability Reduction Model (in our case, the exponent $m$ in Eq. $9 \mathrm{~b}$ is assumed to be 4). Moreover, the flow behavior of the secondary plume strongly depends on the assumptions about the capillary pressures of the grouted sand and the size of the solidified plume which in turn is determined by the parameter $\mathrm{X}_{\min }$ (see Eq. 6). Most of these parameters are not well known and have to be determined by measuring soil characteristics before and after grout emplacement. 


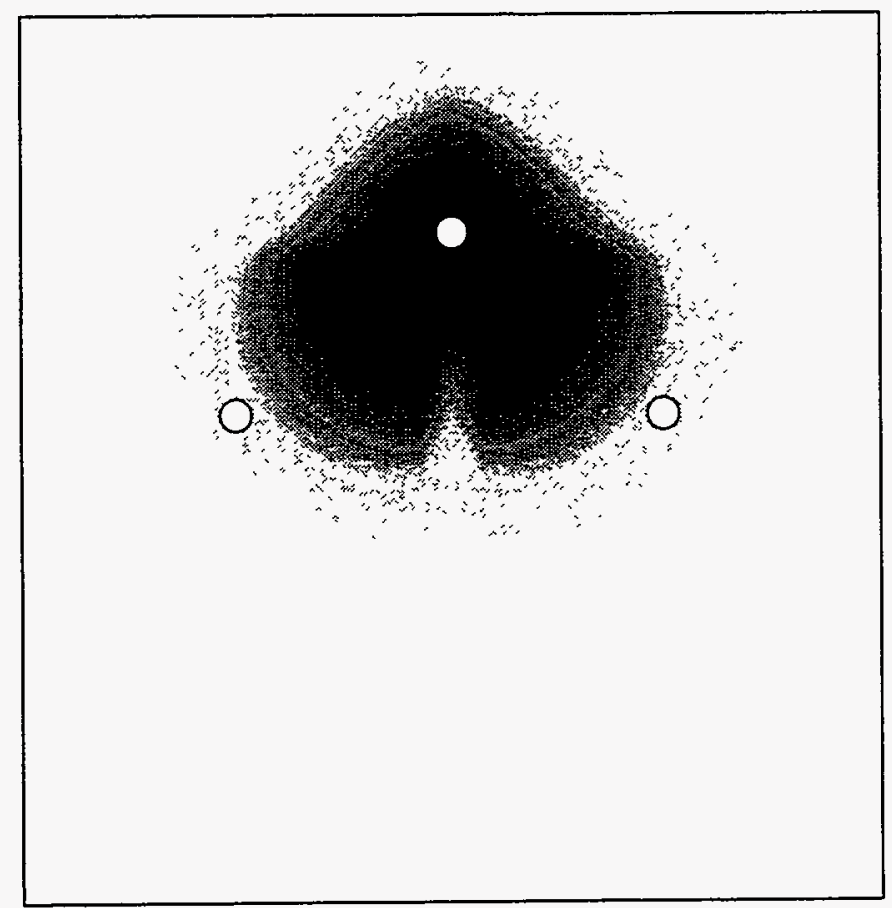

Figure 11: Sample problem 1: Grout content immediately after second injection

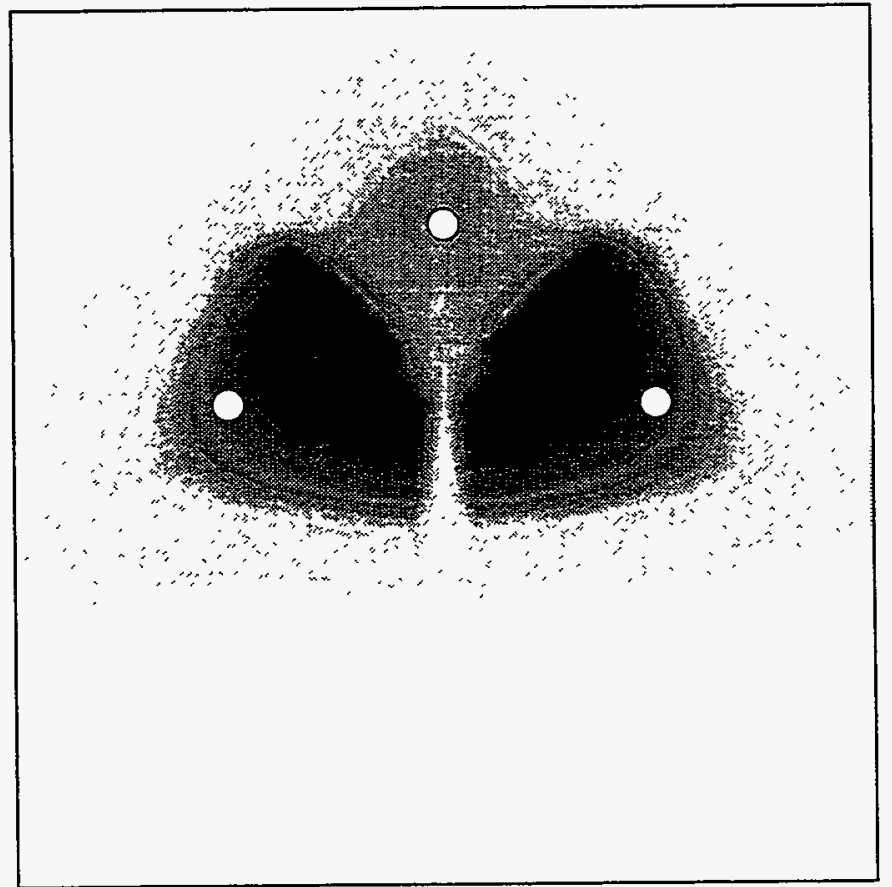

Figure 12: Sample problem 1: Grout content at $t=10$ hours 


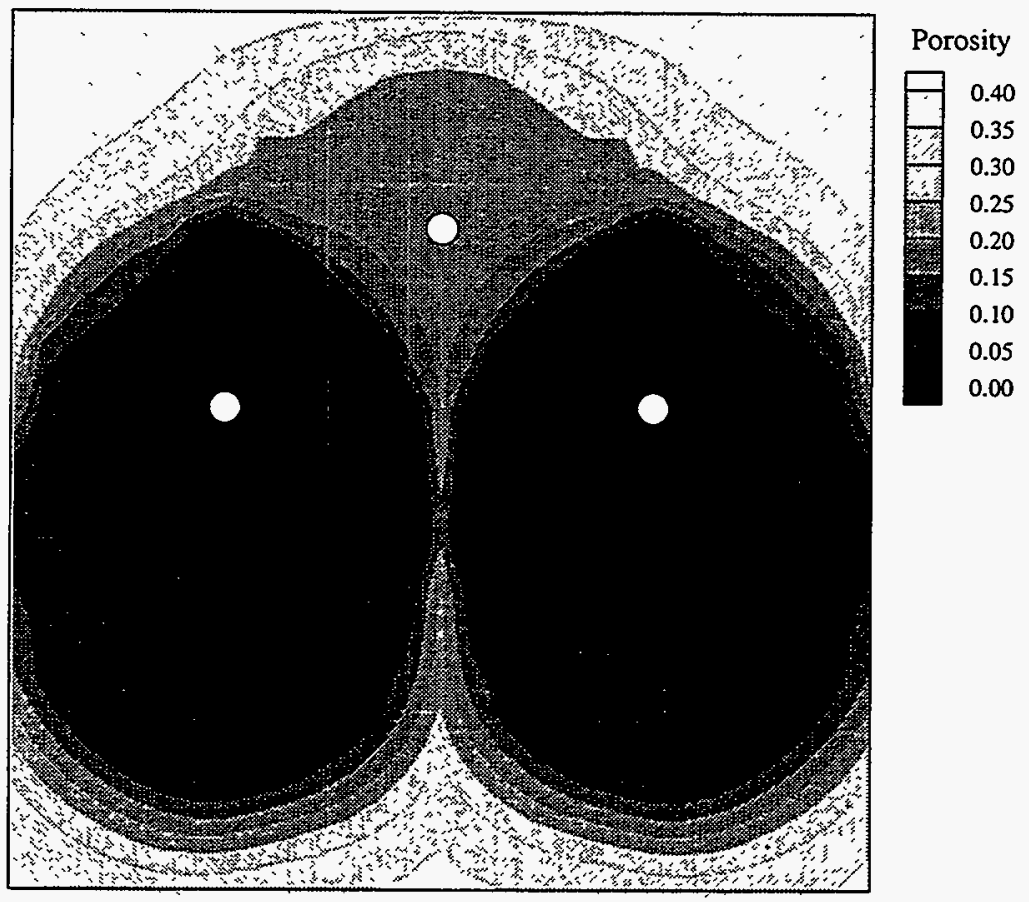

Figure 13: Sample problem 1: Porosity distribution after second solidification

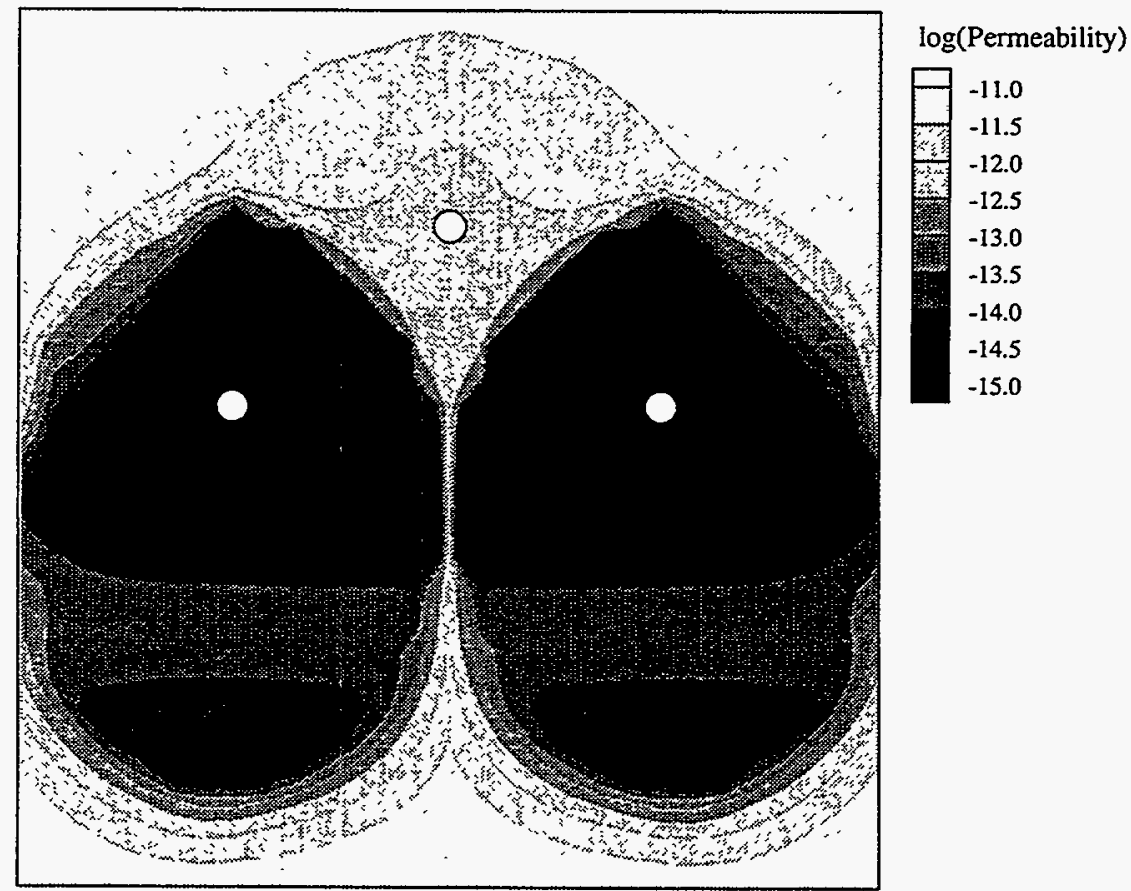

Figure 14: Sample problem 1: Log-Permeability distribution after second solidification 
OUTPUT DATA AETER ( 157,3$)$-2-TIME STEPS

THE TIME IS $.426667 E+00$ DAYS

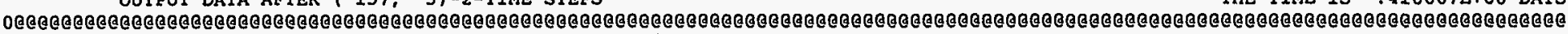

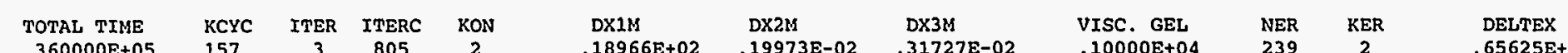

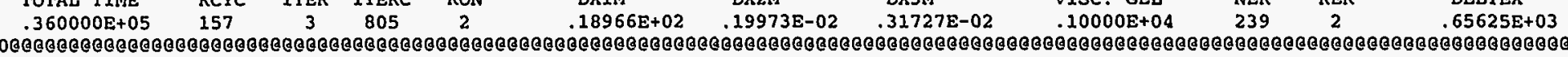

\begin{tabular}{|c|c|c|c|c|c|c|c|c|c|c|c|}
\hline EM. & & $\stackrel{p}{P}$ & $\begin{array}{c}T \\
\text { (DEG-C) }\end{array}$ & SL & XGEL & $\begin{array}{c}\text { VISL } \\
\left(P^{\star}{ }^{\star} S\right)\end{array}$ & OROSITY & $\begin{array}{l}\text { PCAP } \\
\text { (PA) }\end{array}$ & $\begin{array}{c}\text { PERM } \\
\left(M^{\wedge} 2\right)\end{array}$ & $\begin{array}{c}D G \\
\left(K G / M^{\wedge} 3\right)\end{array}$ & $\begin{array}{c}\text { DL } \\
\left(K G / M^{\wedge} 3\right)\end{array}$ \\
\hline & 1 & $.10000 E+06$ & $.20000 \mathrm{E}+02$ & & & & & & & & \\
\hline & 2 & $.10000 \mathrm{E}+06$ & $.20000 \mathrm{E}+02$ & $.30362 E+00$ & $.19090 \mathrm{E}+00$ & $.22690 \mathrm{E}-02$ & $.36000 \mathrm{E}+00$ & $-.53124 E+04$ & $.80000 \mathrm{E}-11$ & $.11777 E+01$ & $.99832 \mathrm{E}+03$ \\
\hline A31 1 & 3 & $.10000 E+06$ & $.20000 \mathrm{E}+02$ & $.31205 \mathrm{E}+00$ & $.47867 E+00$ & $.12093 \mathrm{E}-01$ & $.36000 E+00$ & $-.51564 E+04$ & $.80000 \mathrm{E}-11$ & $.11777 E+01$ & $.99832 E+03$ \\
\hline A41 1 & 4 & $.10000 \mathrm{E}+06$ & $.20000 \mathrm{E}+02$ & $.34555 E+00$ & $.76686 \mathrm{E}+00$ & $.22821 E+00$ & $.36000 E+00$ & $-.46179 E+04$ & $.80000 E-11$ & $.11777 E+01$ & $.99832 \mathrm{E}+03$ \\
\hline A51 1 & 5 & $.10000 E+06$ & $.20000 \mathrm{E}+02$ & $.40217 \mathrm{E}+00$ & $.93452 E+00$ & $.12276 \mathrm{E}+02$ & $.36000 \mathrm{E}+00$ & $-.39251 E+04$ & $.80000 \mathrm{E}-11$ & $.11777 E+01$ & $.99832 E+03$ \\
\hline & 6 & $.10000 \mathrm{E}+06$ & $.20000 \mathrm{E}+02$ & $.41955 \mathrm{E}+00$ & $.98396 \mathrm{E}+00$ & $.20237 E+03$ & $.36000 E+00$ & $-.37523 E+04$ & $.79999 \mathrm{E}-11$ & $.11777 \mathrm{E}+01$ & $.99832 E+03$ \\
\hline & 7 & $.10000 \mathrm{E}+06$ & $.20000 E+02$ & $.42512 \mathrm{E}+00$ & $.99586 \mathrm{E}+00$ & $.62069 \mathrm{E}+03$ & $.35999 E+00$ & $-.37002 E+04$ & $.79990 \mathrm{E}-11$ & $.11777 E+01$ & $.99832 \mathrm{E}+03$ \\
\hline A81 1 & 8 & $.10000 E+06$ & $.20000 \mathrm{E}+02$ & $.42369 \mathrm{E}+00$ & $.99893 \mathrm{E}+00$ & $.87873 E+03$ & $.35989 E+00$ & $-.37140 \mathrm{E}+04$ & $.79905 \mathrm{E}-11$ & $.11777 E+01$ & $.99832 E+03$ \\
\hline A91 1 & 9 & $.10000 \mathrm{E}+06$ & $.20000 E+02$ & $.41718 E+00$ & $.99972 \mathrm{E}+00$ & $.96598 \mathrm{E}+03$ & $.35930 \mathrm{E}+00$ & $-.37788 E+04$ & 83E-11 & $.11777 \mathrm{E}+01$ & $.99832 \mathrm{E}+03$ \\
\hline AA1 1 & 10 & $.10000 \mathrm{E}+06$ & $.20000 \mathrm{E}+02$ & $.40686 \mathrm{E}+00$ & $.99990 \mathrm{E}+00$ & $.98811 \mathrm{E}+03$ & $.35707 E+00$ & $-.38917 E+04$ & $.77424 \mathrm{E}-11$ & $.11777 \mathrm{E}+01$ & $.99832 \mathrm{E}+03$ \\
\hline$A B 11$ & 11 & $.10000 E+06$ & $.20000 E+02$ & $.39378 \mathrm{E}+00$ & $.99962 \mathrm{E}+00$ & $.95457 E+03$ & $.35151 E+00$ & $-.40536 \mathrm{E}+04$ & $.72713 \mathrm{E}-11$ & $.11777 \mathrm{E}+01$ & $.99832 \mathrm{E}+03$ \\
\hline AC1 1 & 12 & $.10000 E+06$ & $.20000 E+02$ & $.37854 E+00$ & $.99631 \varepsilon+00$ & $.65144 E+03$ & $.34146 E+00$ & $-.42683 E+04$ & $47 \mathrm{E}-11$ & $7 E+01$ & $2 \mathrm{E}+03$ \\
\hline AD1 1 & 13 & $.10000 \mathrm{E}+06$ & $.20000 E+02$ & .3613 & $.97636 \mathrm{E}+00$ & $.11330 E+03$ & $.32698 E+00$ & $-.45444 \mathrm{E}+04$ & $.54447 \mathrm{E}-11$ & $.11777 \mathrm{E}+01$ & $.99832 \mathrm{E}+03$ \\
\hline & 14 & $8+06$ & & & & & & & & & \\
\hline AF1 1 & 15 & .1000 & $E+02$ & $E+00$ & $.70066 \mathrm{E}+00$ & EE-01 & $0 E+00$ & $5 E+04$ & $E-11$ & $E+01$ & $2 E+03$ \\
\hline AGI 1 & 16 & & & & & & & -.50 & & & $E+03$ \\
\hline AH1 1 & 17 & $0 \varepsilon+06$ & .20 & .448 & .29671 & & $E+00$ & -.49 & & & \\
\hline AI1 1 & 18 & +06 & .20 & .495 & $.20167 \mathrm{E}+00$ & $E-02$ & $.25383 E+00$ & $-.48106 \mathrm{E}+04$ & $1 E-11$ & $.11777 \mathrm{E}+01$ & $.99832 E+03$ \\
\hline & 19 & & & & & & & & & & \\
\hline AK1 1 & 20 & $8+06$ & .20 & .575 & $E-01$ & $E-02$ & $E+00$ & -.461 & & $E+01$ & $2 E+03$ \\
\hline AL1 1 & 21 & & & & -01 & & +00 & -.45 & -11 & $E+01$ & $8+03$ \\
\hline AM1 1 & 22 & .10 & .20 & .60 & & & & -.44 & & & \\
\hline AN1 1 & 2 & .10 & $0 E+02$ & .57 & 59E-01 & .115 & $77 E+00$ & -.4327 & $E-11$ & $7 E+01$ & $2 E+03$ \\
\hline A01 1 & 24 & & & & & & $E+00$ & -.42 & & & \\
\hline AP1 1 & & .10 & .200 & .468 & .161 & .10 & $E+00$ & -.41 & -11 & $8+01$ & $\varepsilon+03$ \\
\hline$A Q 11$ & & & & & & & $E+\infty$ & -.4 & -11 & & \\
\hline$A R 11$ & 27 & .10 & .20 & .42 & & & & & & & \\
\hline AS1 1 & & & & & & & & -.3 & & & \\
\hline AT1 1 & & & & & & & & & & & \\
\hline AU1 1 & 3 & .1000 & .20 & +00 & $.81016 E-03$ & $.10049 \mathrm{E}-02$ & $.35909 E+00$ & -.36 & $6 E-11$ & $.11777 E+01$ & $.99832 E+03$ \\
\hline A11 2 & 31 & .10 & & & & & $E+00$ & & & & \\
\hline A21 2 & 32 & & .20 & & & & & -.53 & & & $E+03$ \\
\hline A.312 & & .100 & .20 & & $+\infty 0$ & & $0 E+00$ & -.519 & $E-11$ & $E+01$ & $\varepsilon+03$ \\
\hline A11 2 & 34 & & & & & & & & & & \\
\hline A51 2 & 35 & .100 & $E+02$ & $.38524 \mathrm{E}+00$ & .8817 & $.21981 E+01$ & $O E+00$ & -.11 & & $.11777 \mathrm{E}+01$ & $832 E+03$ \\
\hline & 36 & & & & & & & & & & \\
\hline A71 2 & 37 & $.10000 E+06$ & $E+02$ & $.41942 E+00$ & $.98906 \mathrm{E}+00$ & & $.35985 E+00$ & $-.37544 \mathrm{E}+04$ & & $.11777 \mathrm{E}+01$ & $2 E+03$ \\
\hline A81 2 & & & & & & & $.35893 E+00$ & $-.37638 \mathrm{E}+04$ & & $.11777 E+01$ & \\
\hline A91 2 & 39 & $.10000 E+06$ & & & & & & & & & \\
\hline AA1 2 & 10 & & & & & & & & & & \\
\hline AB1 2 & 41 & $.10000 E+06$ & & & & & & $-.41958 \mathrm{E}$ & & & \\
\hline AC1 2 & 42 & $.10000 E+06$ & $000 E+02$ & $.42138 E+00$ & $.99599 E+00$ & $.62910 E+03$ & $.28825 E+00$ & $-.45135 E+04$ & $.32881 \mathrm{E}-11$ & $.11777 E+01$ & $.99832 E+03$ \\
\hline AD1 2 & 4 & & & & & & & -.498371 & & & \\
\hline & 44 & & & & & & & & & & \\
\hline AF1 2 & 45 & $.10292 \mathrm{E}+06$ & $8+02$ & & & & & & & & \\
\hline
\end{tabular}


1Sample Problem 1: Multiple Grout Injections Into Unsaturated sand

OUTPUT DATA AFTER $(157,3)-2$-TIME STEPS

THE TIME IS .416667 E +00 DAYS

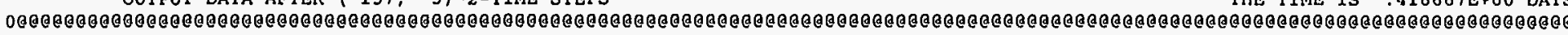

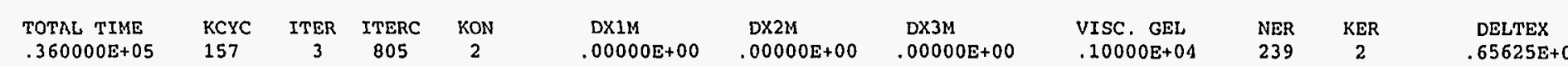

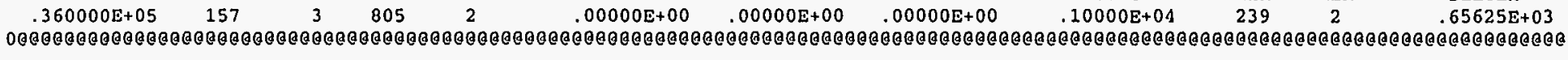

\begin{tabular}{|c|c|c|c|c|c|c|c|c|c|c|c|}
\hline OELEM. & INDEX & $\begin{array}{c}P \\
(P A)\end{array}$ & $\begin{array}{c}\mathrm{T} \\
(D E G-C)\end{array}$ & SL & XGEL & $\begin{array}{c}\text { VISL } \\
\left(P A^{*} S\right)\end{array}$ & POROSITY & $\begin{array}{l}\text { PCAP } \\
\text { (PA) }\end{array}$ & $\begin{array}{c}\text { PERM } \\
\left(M^{\wedge} 2\right)\end{array}$ & $\begin{array}{c}D G \\
\left(K G / M^{\wedge} 3\right)\end{array}$ & $\begin{array}{c}D L \\
\left(K G / M^{\wedge} 3\right)\end{array}$ \\
\hline A11 1 & 1 & & & & & & & & $.80000 \mathrm{E}-11$ & $.11777 \mathrm{E}+01$ & $.99832 \mathrm{E}+03$ \\
\hline A21 1 & 2 & $.10000 E+06$ & $20000 \mathrm{E}+02$ & $.23435 \mathrm{E}-01$ & $.10507 \mathrm{E}-01$ & $.22690 \mathrm{E}-02$ & $.25671 \mathrm{E}+00$ & $-.53124 E+04$ & $.20686 \mathrm{E}-11$ & $.11777 \mathrm{E}+01$ & $.99832 \mathrm{E}+03$ \\
\hline A31 1 & 3 & $.10000 \mathrm{E}+06$ & $.20000 \varepsilon+02$ & $.45155 \mathrm{E}-02$ & $.47867 \mathrm{E}-02$ & $.12093 \mathrm{E}-01$ & $.24878 \mathrm{E}+00$ & $-.51564 \mathrm{E}+04$ & $.18246 \mathrm{E}-11$ & $.11777 E+01$ & $.99832 E+03$ \\
\hline A41 1 & 4 & $.10000 \mathrm{E}+06$ & $.20000 \mathrm{E}+02$ & $.52523 \mathrm{E}-02$ & $.76686 \mathrm{E}-02$ & $.22821 \mathrm{E}+00$ & $.23685 E+00$ & $-.46179 E+04$ & $.14988 \mathrm{E}-11$ & $.11777 \mathrm{E}+01$ & $.99832 \mathrm{E}+03$ \\
\hline A51 1 & 5 & $.10000 \mathrm{E}+06$ & $.20000 \mathrm{E}+02$ & $.66823 \mathrm{E}-02$ & $.93452 \mathrm{E}-02$ & $.12276 \mathrm{E}+02$ & $.21667 \mathrm{E}+00$ & $-.39251 \mathrm{E}+04$ & $.10496 \mathrm{E}-11$ & $.117778+01$ & $.99832 \mathrm{E}+03$ \\
\hline A61 1 & 6 & $.10000 \mathrm{E}+06$ & $.20000 \mathrm{E}+02$ & $.71763 \mathrm{E}-02$ & $.98396 \mathrm{E}-02$ & $.20237 E+03$ & $.21047 \mathrm{E}+00$ & $-.37523 \mathrm{E}+04$ & $.93465 \mathrm{E}-12$ & $.11777 E+01$ & $.99832 \mathrm{E}+03$ \\
\hline A71 1 & 7 & $.10000 \mathrm{E}+06$ & $.20000 E+02$ & $.73406 \mathrm{E}-02$ & $.99586 \mathrm{E}-02$ & $.62069 \mathrm{E}+03$ & $.20848 \mathrm{E}+00$ & $-.37002 E+04$ & $.89980 \mathrm{E}-12$ & $.11777 E+01$ & $.99832 \mathrm{E}+03$ \\
\hline A81 1 & 8 & $.10000 \mathrm{E}+06$ & $.20000 \mathrm{E}+02$ & $.72982 \mathrm{E}-02$ & $.99893 \mathrm{E}-02$ & $.87873 E+03$ & $.20893 E+00$ & $-.37140 \mathrm{E}+04$ & $.90769 \mathrm{E}-12$ & $.11777 E+01$ & $.99832 \mathrm{E}+03$ \\
\hline A91 1 & 9 & $.10000 \mathrm{E}+06$ & $.20000 \mathrm{E}+02$ & $.71070 \mathrm{E}-02$ & $.99972 \mathrm{E}-02$ & $.96598 \mathrm{E}+03$ & $.21091 \mathrm{E}+00$ & $-.37788 E+04$ & $.94243 \mathrm{E}-12$ & $.11777 E+01$ & \\
\hline AA1 1 & 10 & $.10000 \mathrm{E}+06$ & $.20000 \mathrm{E}+02$ & $.68128 \mathrm{E}-02$ & $.99990 \mathrm{E}-02$ & $.98811 \mathrm{E}+03$ & $.21324 \mathrm{E}+00$ & $-.38917 E+04$ & $.98480 \mathrm{E}-12$ & $.11777 \mathrm{E}+01$ & $.99832 \mathrm{E}+03$ \\
\hline$A B 11$ & 11 & $.10000 E+06$ & $.20000 E+02$ & $.64537 \mathrm{E}-02$ & $.99962 \mathrm{E}-02$ & $.95457 \mathrm{E}+03$ & $.21447 E+00$ & $-.40536 E+04$ & $.10078 \mathrm{E}-11$ & $.11777 \mathrm{E}+01$ & $.99832 \mathrm{E}+03$ \\
\hline$A C 1$ & 12 & $.10000 \mathrm{E}+06$ & $.20000 E+02$ & $.60542 \mathrm{E}-02$ & $.99631 \mathrm{E}-02$ & $.65144 \mathrm{E}+03$ & $.21350 \mathrm{E}+00$ & $-.42683 E+04$ & $.98958 \mathrm{E}-12$ & $.11777 E+01$ & $.99832 \mathrm{E}+03$ \\
\hline$A D 1$ & 13 & $.10000 \mathrm{E}+06$ & $.2,0000 \mathrm{E}+02$ & $.56254 \mathrm{E}-02$ & $.97636 \mathrm{E}-02$ & $.11330 E+03$ & $.21002 \mathrm{E}+00$ & $-.45444 E+04$ & $.92670 \mathrm{E}-12$ & $.11777 \mathrm{E}+01$ & $.99832 \mathrm{E}+03$ \\
\hline AE1 1 & 14 & $.10000 \mathrm{E}+06$ & $.20000 \mathrm{E}+02$ & $.52271 \mathrm{E}-02$ & $.89587 \mathrm{E}-02$ & $.32526 \mathrm{E}+01$ & $.20387 E+00$ & $-.48701 \mathrm{E}+04$ & & $.11777 E+01$ & $.99832 E+03$ \\
\hline AFI 1 & 15 & $.10000 \mathrm{E}+06$ & $.20000 \mathrm{E}+02$ & $.51520 \mathrm{E}-02$ & $.70066 \mathrm{E}-02$ & $.93752 \mathrm{E}-01$ & $.19251 \mathrm{E}+00$ & $-.51255 E+04$ & $.65410 \mathrm{E}-12$ & $.11777 \varepsilon+01$ & $.99832 \mathrm{E}+03$ \\
\hline AG1 1 & 16 & $.10000 \mathrm{E}+06$ & $.20000 \mathrm{E}+02$ & $.63480 \mathrm{E}-02$ & $.46672 E-02$ & .1110 & $.16798 \mathrm{E}+00$ & $-.50151 E+04$ & $.37924 \mathrm{E}$ & $.11777 E+01$ & $.99832 E+03$ \\
\hline AHI 1 & 17 & .100 & .200 & .80656 & & & & -.491 & & & $E+03$ \\
\hline AI1 1 & 18 & .1000 & .2000 & .97216 & $.20167 E-02$ & .238 & $.12934 E+00$ & -.481 & $0 E-12$ & $.11777 E+01$ & $.99832 E+03$ \\
\hline AJI 1 & 19 & $.10000 \mathrm{E}+06$ & $.20000 \mathrm{E}+02$ & & $.47738 \mathrm{E}-01$ & & $.16718 \mathrm{E}+00$ & $-.47117 \mathrm{E}+04$ & & & \\
\hline AK1 1 & 20 & $.10000 \mathrm{E}+06$ & $.20000 \mathrm{E}+02$ & $.47749 E+00$ & $.44409 \mathrm{E}-01$ & $.13042 \mathrm{E}-02$ & $.20021 \mathrm{E}+00$ & $-.46147 \mathrm{E}+04$ & $.76537 \mathrm{E}-12$ & $77 E+01$ & $.99832 \mathrm{E}+03$ \\
\hline AL1 1 & & $0 \mathrm{E}+06$ & $00 \mathrm{E}+02$ & .53979 & $99 E-01$ & .1208 & $0 \mathrm{E}+00$ & -.451 & & $.11777 E+01$ & $.99832 \mathrm{E}+03$ \\
\hline AM1 1 & 22 & .10000 & .2000 & $E+00$ & $.32569 \mathrm{E}-01$ & .117 & $.21760 \mathrm{E}+00$ & $-.44236 E+04$ & & $.11777 E+01$ & $.99832 \mathrm{E}+03$ \\
\hline AN1 1 & 2. & $.10000 \mathrm{E}+06$ & $.20000 \mathrm{E}+02$ & $.52771 \mathrm{E}+00$ & $.29501 \mathrm{E}-01$ & $E-02$ & $.23051 E+00$ & $-.43279 \mathrm{E}+04$ & $5-11$ & $.11777 E+01$ & $.99832 \mathrm{E}+03$ \\
\hline $\mathrm{AO1} 1$ & 24 & .1000 & & & & & & -.42 & & & $E+03$ \\
\hline AP1 1 & 25 & $.10001 E+06$ & $.20000 \mathrm{E}+02$ & $.44739 E+00$ & $.14830 \mathrm{E}-01$ & .10668 & $.28145 E+00$ & -.413 & .298 & $.11777 \mathrm{E}+01$ & $2 E+03$ \\
\hline$A Q 1$ & & .1000 & $.20000 \mathrm{E}+02$ & $.42246 E+00$ & $.93546 \mathrm{E}-02$ & & $.30743 E+00$ & -.403 & & $.11777 \mathrm{E}$ & \\
\hline AR1 1 & 27 & & & & & & & -.39 & & & \\
\hline AS1 1 & 28 & $.10001 \mathrm{E}+06$ & $.20000 \mathrm{E}+02$ & .41389 & $.33806 \mathrm{E}-02$ & .1015 & $.34516 E+00$ & $-.38439 E+04$ & .6760 & $.11777 \mathrm{E}+01$ & $2 \mathrm{E}+03$ \\
\hline AT1 1 & & .100 & .200 & .42 & .182 & & .35 & -.37 & & & $E+03$ \\
\hline AU1 1 & 30 & .10001 & $.2000 \mathrm{C}$ & & & & $.35847 E+00$ & -.364 & .786 & .117 & $E+03$ \\
\hline A11 2 & 31 & .10000 & .20000 & .1000 & $.00000 \mathrm{E}+00$ & -02 & $.36000 E+00$ & .000 & & & \\
\hline A21 2 & 32 & & & & & & & $-.53373 E+04$ & & & \\
\hline A31 2 & 33 & $.10000 \mathrm{E}+06$ & $.20000 \mathrm{E}+02$ & $.44672 \mathrm{E}-02$ & $.41880 \mathrm{E}-02$ & $.80222 \mathrm{E}-02$ & $.24961 E+00$ & $-.51983 E+04$ & $.18490 \mathrm{E}-11$ & $.11777 \mathrm{E}+01$ & $E+03$ \\
\hline A41 2 & & .10000 & & & & & & -.48 & & & \\
\hline A51 2 & 35 & $.10000 \mathrm{E}+06$ & $.20000 \mathrm{E}+02$ & .62275 & & .21981 & $.22270 \mathrm{E}+00$ & $-.41095 \mathrm{E}+04$ & .1171 & $.11777 \mathrm{E}+01$ & $.99832 \mathrm{E}+03$ \\
\hline A61 2 & 36 & $.10000 \mathrm{E}+06$ & & & & & & $-.38284 E+04$ & & $.11777 E+01$ & $.99832 E+03$ \\
\hline A71 2 & 37 & $.10000 \mathrm{E}+06$ & & & & & & $-.37544 \mathrm{E}+04$ & & & \\
\hline A81 2 & 38 & $.10000 E+06$ & & & & & & $-.37638 E+04$ & & .1177 & \\
\hline A91 & 3 & & & & & & & & & & \\
\hline AA1 2 & 40 & $.10000 \mathrm{E}+06$ & & & $.99966 \mathrm{E}-02$ & & $.20510 \mathrm{E}+00$ & $-.39779 E+04$ & & $.11777 \mathrm{E}+01$ & \\
\hline$A B 12$ & 41 & & & & & & & $-.41958 \mathrm{E}+04$ & & $.11777 \mathrm{E}+01$ & \\
\hline $\mathrm{AC} 12$ & 42 & $00 \mathrm{E}+06$ & & & & $.62910 \mathrm{E}+03$ & $.16800 \mathrm{E}+00$ & & & $.11777 \mathrm{E}$ & \\
\hline & & & & & $114 \mathrm{E}-02$ & $.16162 \mathrm{E}+03$ & $.12244 \mathrm{E}+00$ & & & & \\
\hline AE 1 & 44 & & & & & & & & & & \\
\hline AF1 2 & 45 & $.10292 E+06$ & $.20000 \mathrm{E}+02$ & $.84374 E+00$ & $.85443 \mathrm{E}-02$ & $.11287 E+01$ & $.18512 \mathrm{E}-02$ & $-.80460 \mathrm{E}+04$ & $.55939 \mathrm{E}-20$ & $.12124 \mathrm{E}+01$ & $.99832 E+03$ \\
\hline
\end{tabular}




\subsection{Sample 2: Horizontal Barrier Emplacement}

The second sample problem considers the emplacement of a horizontal subsurface barrier beneath a rectangular slurry wall container of $10 \times 16$ feet. Grout is injected in the saturated zone from two horizontal wells on one side of the slurry wall. Water is pumped from two extraction wells on the other side of the container to induce a groundwater current which enables the emplacement of a horizontal grout floor. Injection and production occurs at a constant rate of $0.1 \mathrm{~kg} / \mathrm{sec}$ in each well for 3 days. Two circulation wells are installed within the slurry wall system to prevent the grout from mounding up into the container. The Gel Time Curve of Figure 1 has been stretched in time by a factor of 60 (see parameter FE(18)).

A heterogeneous, anisotropic permeability field has been generated using simulated annealing techniques. The mean of the permeability field is $1.0 \mathrm{E}-11 \mathrm{~m}^{2}$. The standard deviation of the logarithm is 1.0 . The generated permeability field follows a spherical variogram with a horizontal and vertical correlation length of 3.0 and 0.5 meters, respectively. The TOUGH2 input file is shown in Figure 17; the permeability field and the experimental layout are illustrated in Figure 18.

Figures 19 through 22 show grout concentrations at different times. After three days of pumping, the pressure in the injection wells starts to increase due to the gelation of the grout. These figures show that the grout plume has successfully been pulled to the other side of the slurry wall container. Injection stops and the Solidification Model is applied. The final permeability field of the grouted aquifer is shown in Figure 23. 


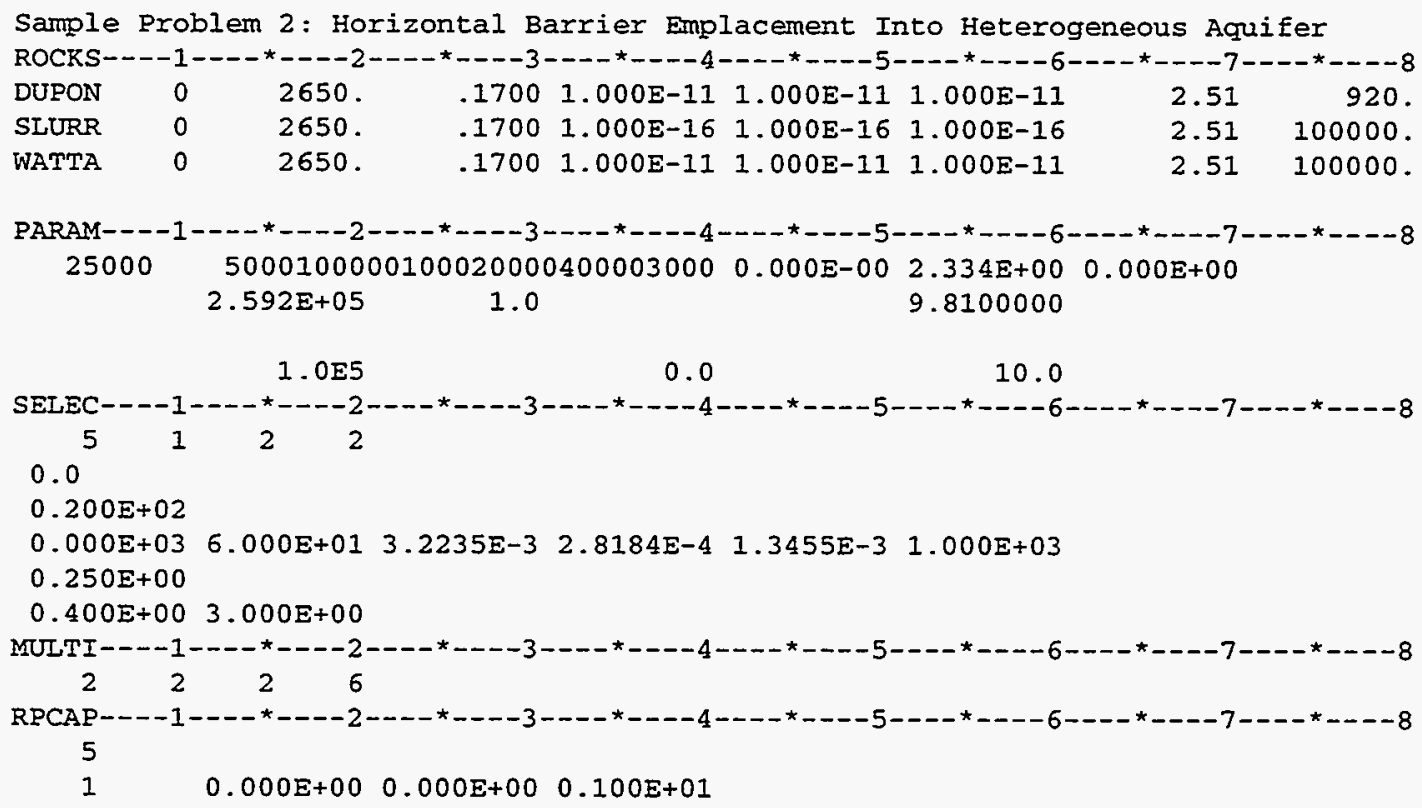

INCON Spatially correlated random permeability field generated -*..--7-..-*..--8 A11 $1 \quad .17000000 E+00 \quad .6674 E-11$ $.100000000000 \mathrm{E}+06 \quad .000000000000 \mathrm{E}+00 \quad .100000000000 \mathrm{E}+02$

Figure 17: TOUGH2 input file for sample problem No. 2 


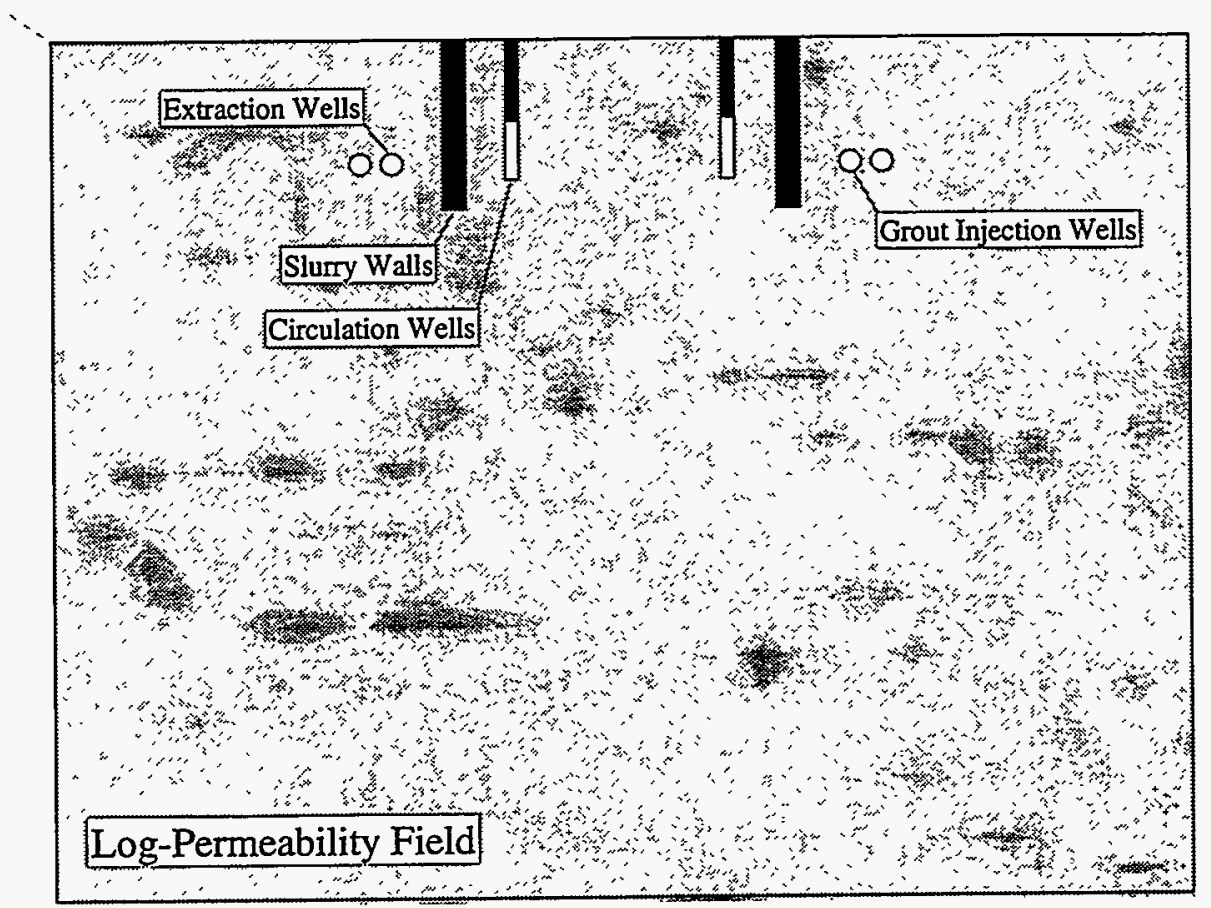

Figure 18: Sample problem 2: Permeability distribution 


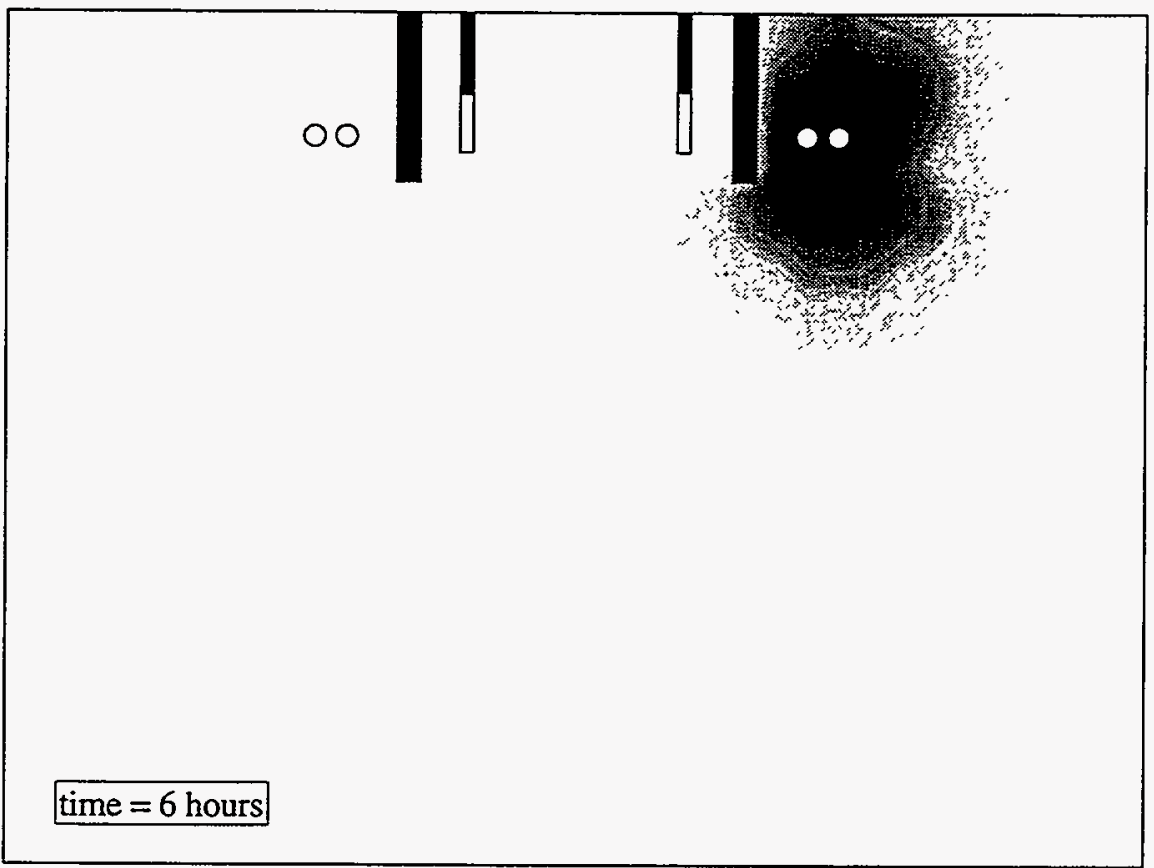

Figure 19: Sample problem 2: Grout concentration after 6 hours

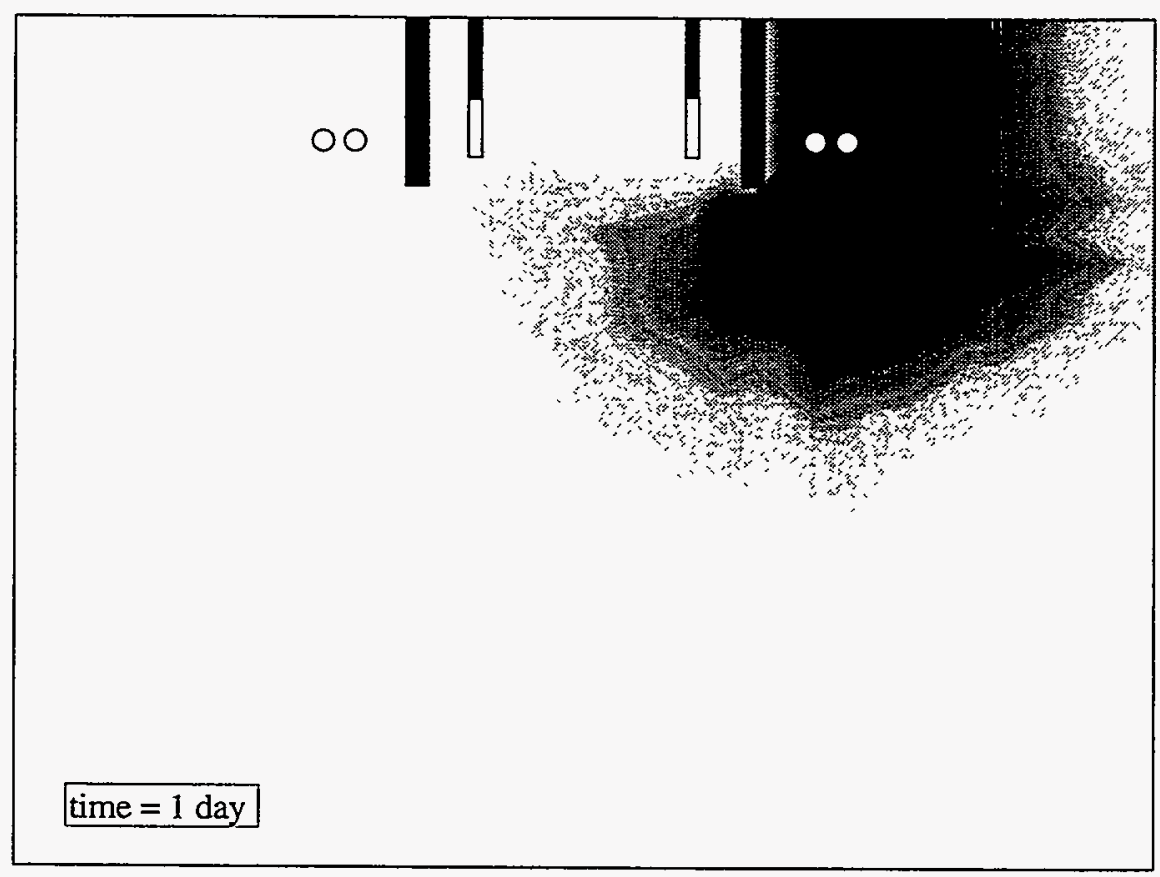

Figure 20: Sample problem 2: Grout concentration after 1 day 


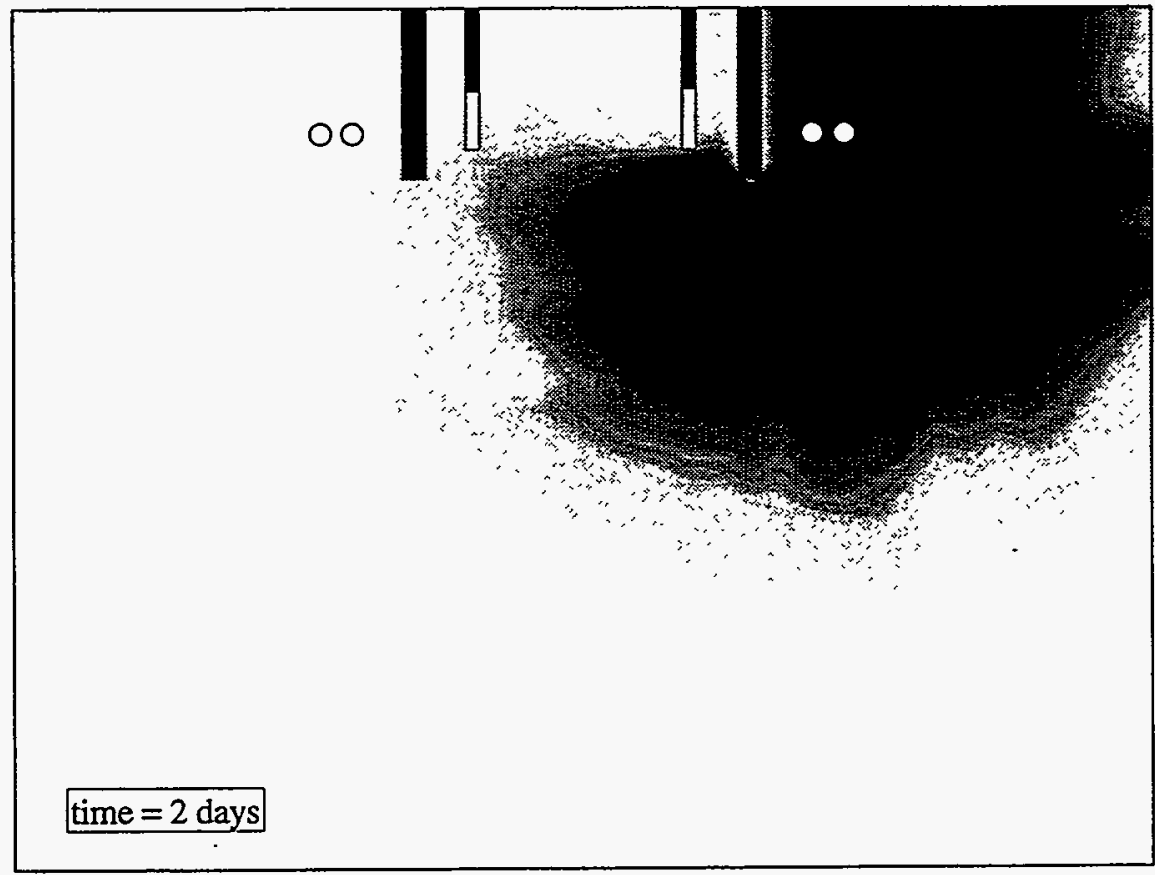

Figure 21: Sample problem 2: Grout concentration after 2 days

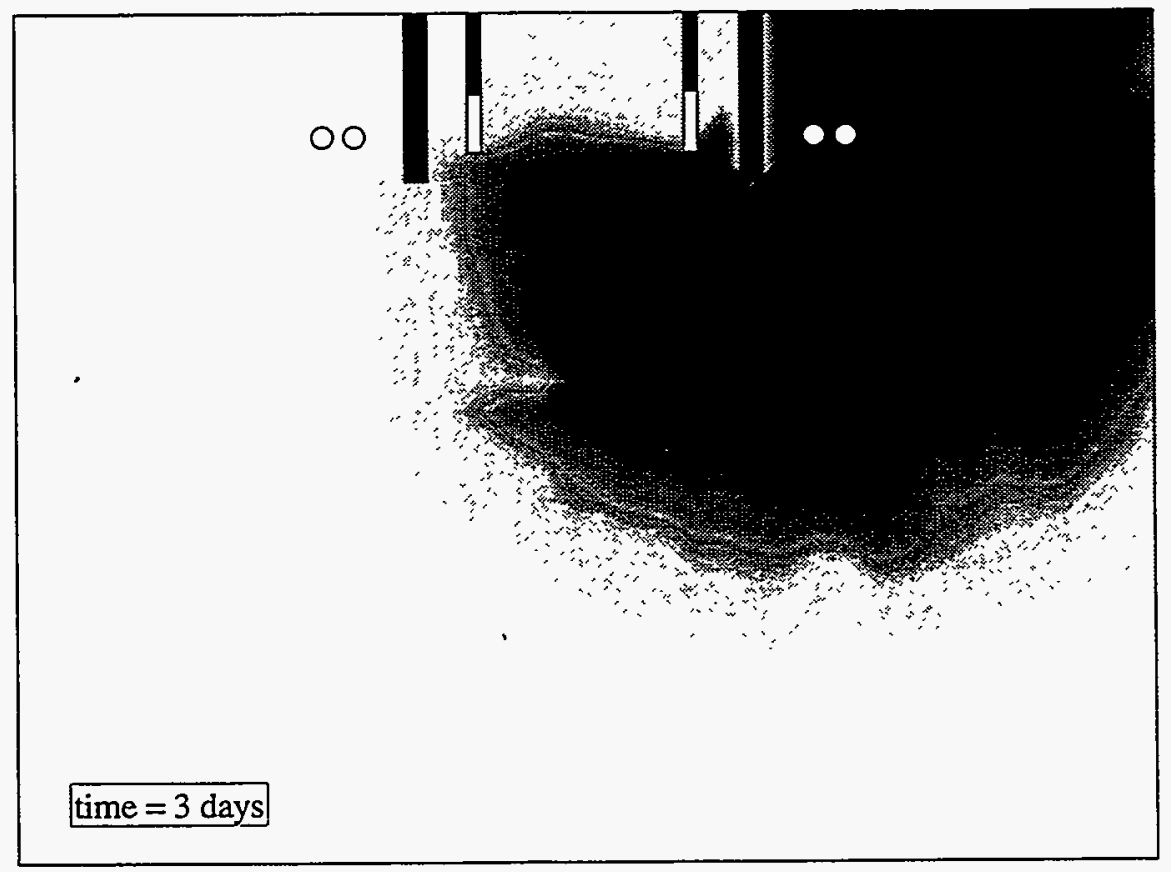

Figure 22: Sample problem 2: Grout concentration after 3 days 


\section{ภิụฺno.ภ̊}

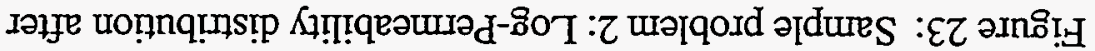

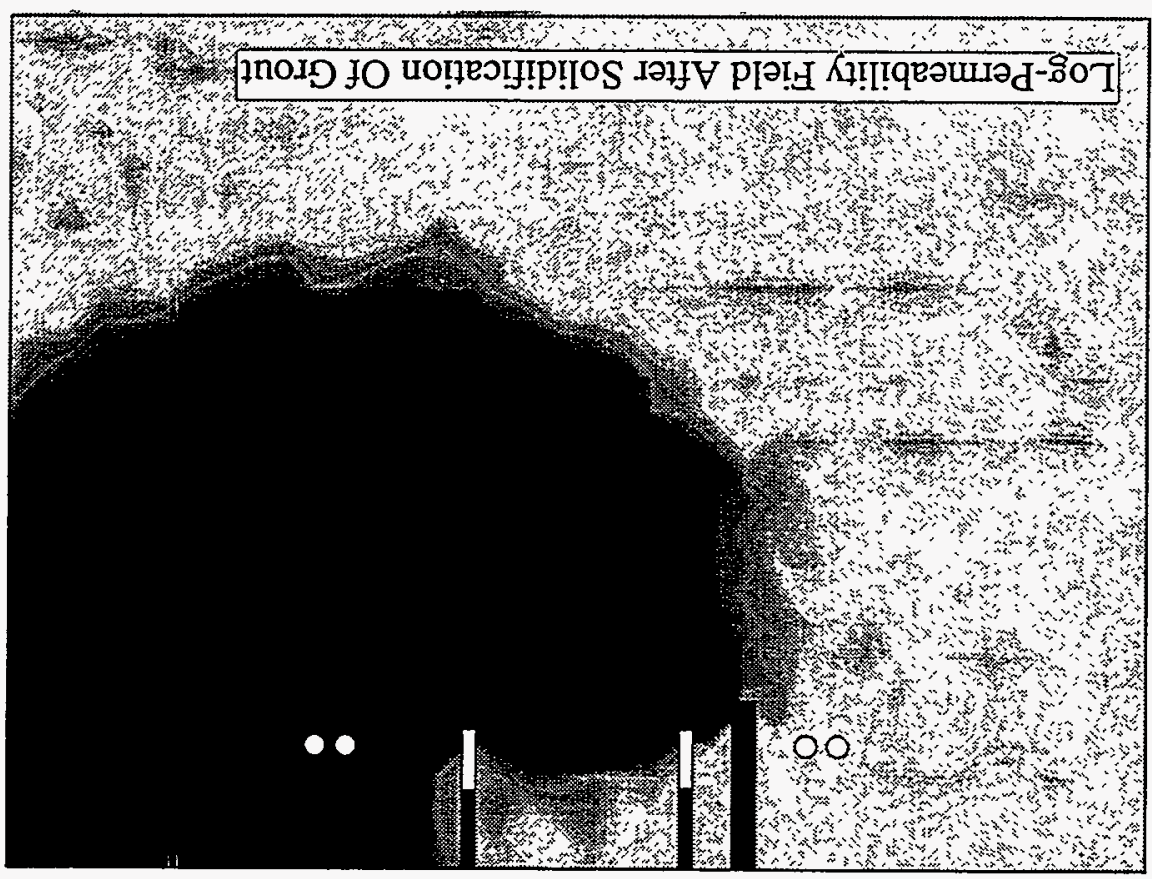




\section{ACKNOWLEDGMENT}

This work is supported by the In Situ Remediation Technology Development Integrated Program, Office of Technology Development, Office of Environmental Restoration and Waste Management, U.S. Department of Energy, under contract DE-AC03-76SF00098. We would like to thank A. Datta Gupta for providing a code for the generation of spatially correlated random permeability fields. Thanks are due to E. Antunez and C. Oldenburg for a careful review of the manuscript.

\section{REFERENCES}

Brooks, R. H., and A. T. Corey, Hydraulic properties of porous media, Hydrol. Pap., Colorado State Univ., Fort Collins, CO, 3, 1-27, 1964.

Burdine, N. T., Relative permeability calculations from pore-size distribution data, Trans. Am. Inst. Min. Metall. Pet. Eng., 198, 71-78, 1953.

Finsterle, S., ITOUGH2 User's Guide, Lawrence Berkeley Laboratory Report LBL-34581, Berkeley, CA, 1993.

Finsterle, S., G. J. Moridis, K. Pruess, A TOUGH2 Equation-of-State Module for the Simulation of Two-Phase Flow of Air, Water, and an Immiscible Gelling Liquid, Lawrence Berkeley Laboratory Report, Berkeley, CA, 1994 (in preparation).

Hortes, E., Development of a reservoir model for polymer/gel treatments, Report 87-3, University of Texas at Austin, 1986.

Iler, R. K., The chemistry of silica, Wiley-Interscience, New York, 1979.

International Formulation Committee, A formulation of the thermodynamic properties of ordinary water substance, IFC Secretariat, Düsseldorf, Germany, 1967.

Luckner, L., M. Th. van Genuchten, and D. Nielsen, A consistent set of parametric models for the two-phase flow of immiscible fluids in the subsurface, Water Resour. Res., 25 (10), 2187-2193, 1989. 
McCool, C. S., An experimental study of the in situ gelation Polyacrylamide/Chromium(III)/ Thiourea system in a porous medium, Ph.D. dissertation, University of Kansas, 1988.

Moridis, G. J., P. Persoff, H.-Y. Holman, S. J. Muller, K. Pruess, P. Witherspoon, and C. J. Radke, Containment of contaminants through physical barriers formed from viscous liquids emplaced under controlled viscosity conditions, FY 1993 Annual Report, Lawrence Berkeley Laboratory Report LBL-29400, Berkeley, CA, 1994.

Mualem, Y., A new model for predicting the hydraulic conductivity of unsaturated porous media, Water Resour. Res., 12 (3), 513-522, 1978.

Oldenburg, C. M., and F. J. Spera, Hybrid model for solidification and convection, Numerical Heat Transfer, Part B, vol. 21, 217-229, 1992.

Pruess, K, TOUGH user's guide, Lawrence Berkeley Laboratory Report LBL-20700, Berkeley, CA, 1987.

Pruess, K, TOUGH2 - A general-purpose numerical simulator for multiphase fluid and heat flow, Lawrence Berkeley Laboratory Report LBL-29400, Berkeley, CA, 1991a.

Pruess, K., EOS7, An equation-of-state module for the TOUGH2 simulator for two-Phase flow of saline water and air, Lawrence Berkeley Laboratory Report LBL-31114, Berkeley, CA, $1991 \mathrm{~b}$.

Scott, T., L. J. Roberts and S. R. Short, In situ gel calculations in complex reservoir systems using a new chemical flood simulator, SPE \#14234, presented at the Annual Technical Conference at Las Vegas, NV, September 22-25, 1985.

Todd, B. J., Numerical modeling of in situ gelation in porous media, Ph.D. dissertation, Dept. of Chem. and Petr. Eng., Univ. of Kansas, 1990.

van Genuchten, M. Th., A closed-form equation for predicting the hydraulic conductivity of unsaturated soils, Soil Sci. Soc. Am. J.,44, 892-898, 1980. 


\section{APPENDIX A: SUbroutine GELLING}

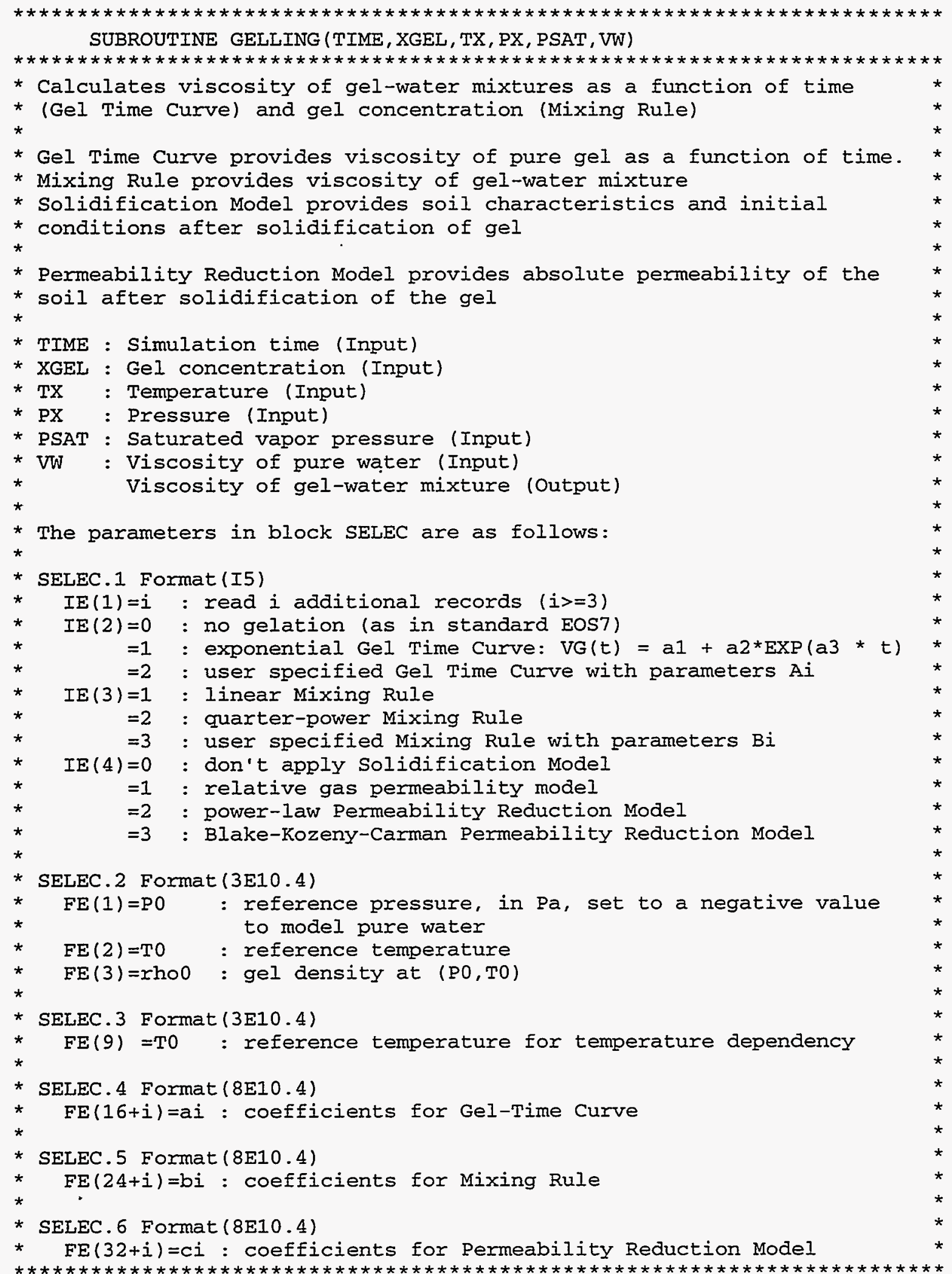


SAVE ICALI

COMMON/NN / NEL , NCON , NOGN , NK, NEQ , NPH , NB , NK1 , NEQ1 , NBK, NSEC , NFLUX COMMON/CYC/KCYC, ITER, ITERC, TIMIN, SUMTIM, GF , TIMOUT COMMON/EOSEL/IE (16), FE(512)

DATA ITEROLD/-1/

DATA ICALL/0/

$I C A L I=I C A L L+1$

IF (ICALL.EQ.1) WRITE (11,899)

899 FORMAT(/6X, 'GELLING 0.5 GEL 25 APRIL 1994',6X, X'GEL TIME CURVE AND MIXING RULE')

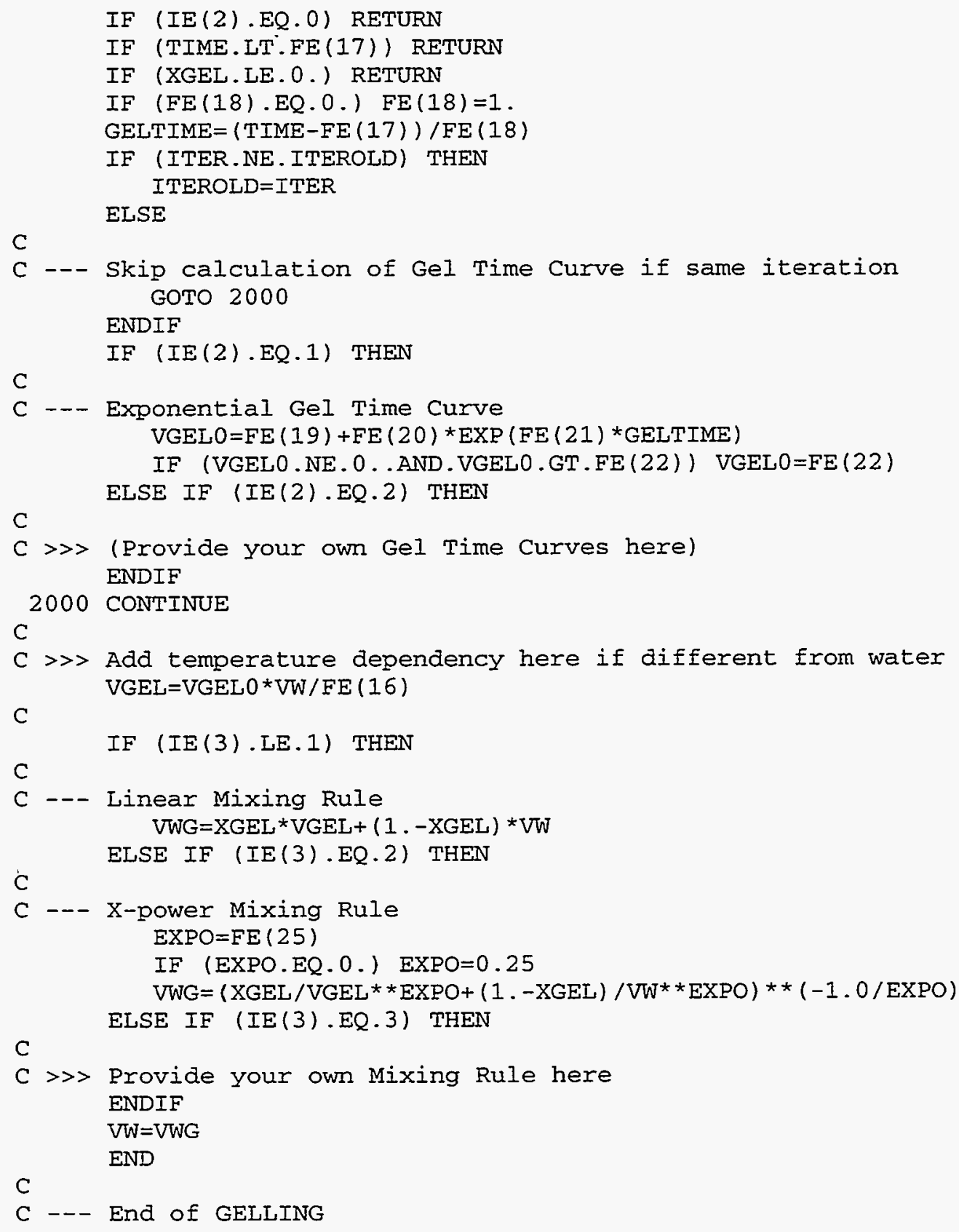




\section{APPENDIX B： PROGRAM SOLIMOD}

Program SOLIMOD is a postprocessor which calculates initial conditions and soil properties of grouted porous media after solidification of the gel. Input to SOLIMOD is a TOUGH2 file SAVE; output of SOLIMOD is a TOUGH2 file INCON.

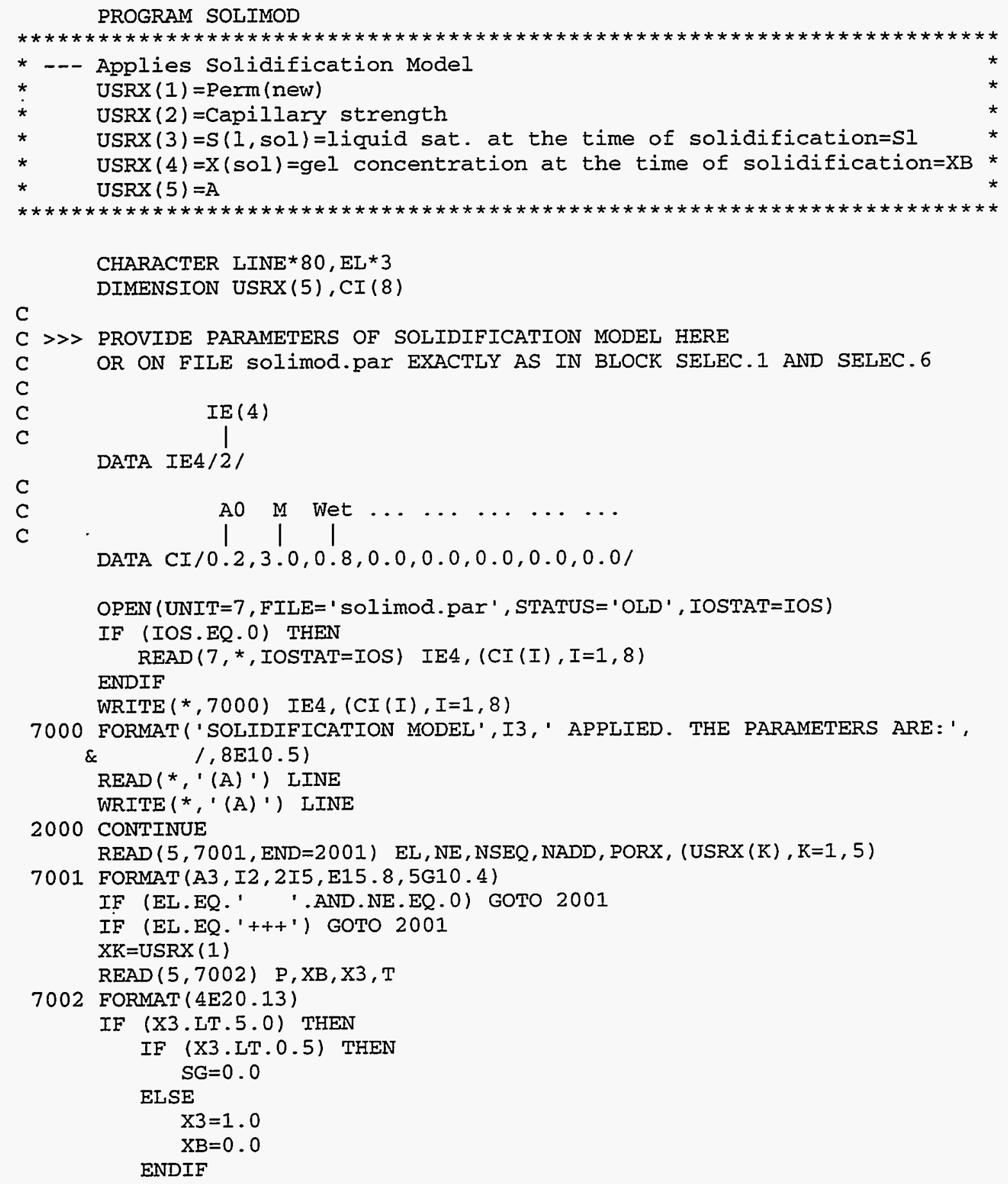




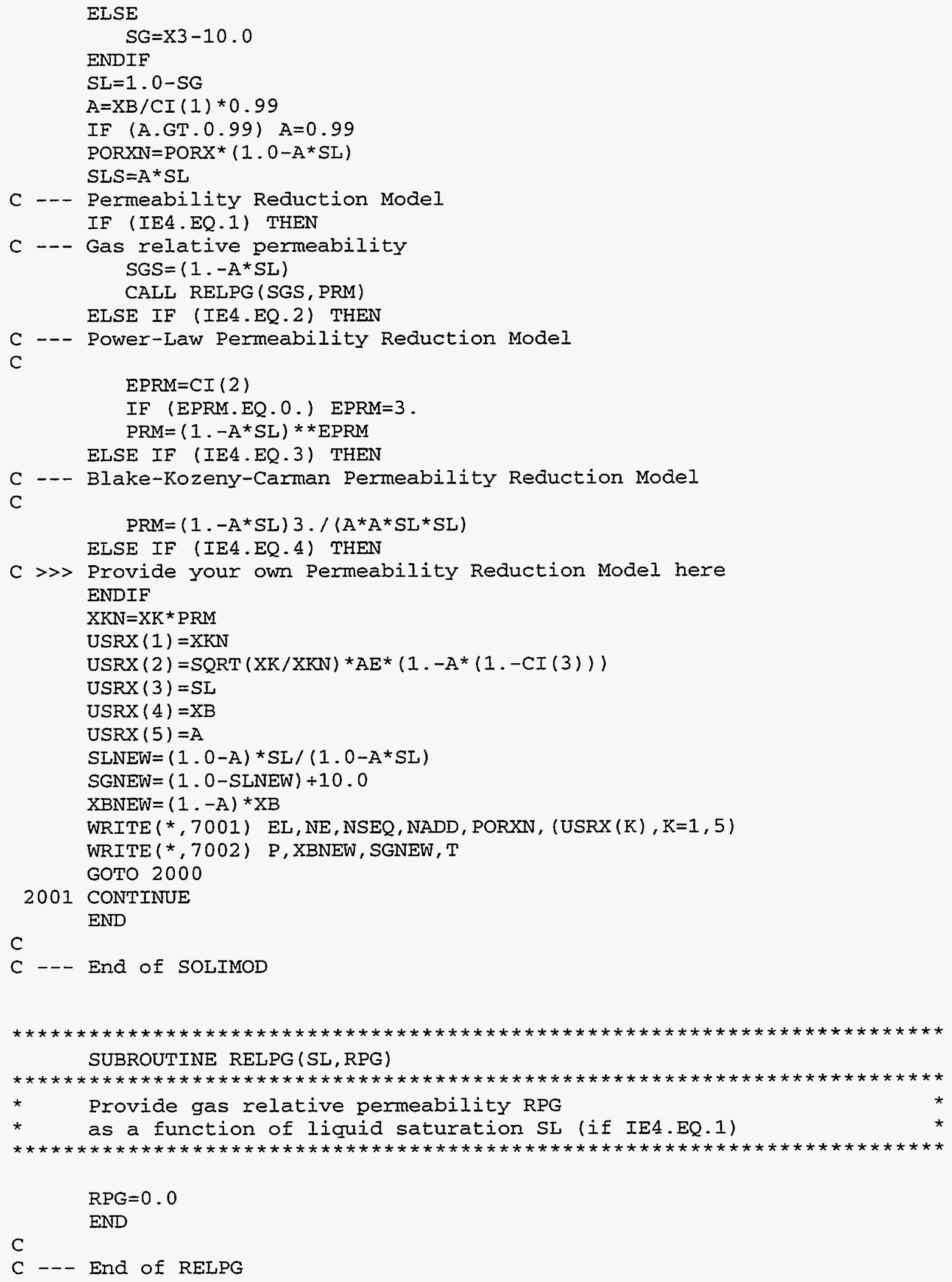

